Chem. Ber. 118, 4203-4223 (1985)

\title{
Versuche zur Synthese eines formalen Nitriloxid-Addukts des Benzols 1,3-Dipolare Cycloadditionen an Bicyclo[2.2.0]hex-5-en-2,3- dicarbonsäureanhydrid, Dewarbenzol und 1,4-Cyclohexadien
}

\author{
Manfred Christl* und Brigitte Mattauch ${ }^{1)}$
}

Institut für Organische Chemie der Universität Würzburg, Am Hubland, D-8700 Würzburg

Eingegangen am 21. Dezember 1984

Aus Bicyclo[2.2.0]hex-5-en-2,3-dicarbonsäureanhydrid (7) wurden durch Reaktion mit Benzonitriloxid, 2,4,6-Trimethylbenzonitriloxid, Diphenylnitrilimin, Diazomethan, Tetrachlor-ochinon und Tetrachlorthiophen-1,1-dioxid die $\Delta^{2}$-Isoxazoline 8a, 8b, das $\Delta^{2}$-Pyrazolin $8 c$, das $\Delta^{1}$-Pyrazolin 10d, das $\alpha$-Diketon 11 bzw. das Tetrachlor-1,3-cyclohexadien-Derivat 12 erhalten. - Durch Anlagerung von 4-Methyl-4H-1,2,4-triazol-3,5-dion an Benzvalen zum Urazol 13, dessen Umwandlung in die Azoverbindung 17 und deren Photolyse wurde Dewarbenzol (16) dargestellt. Seine Reaktionen mit Benzo- und 2,4,6-Trimethylbenzonitriloxid sowie Diazomethan erbrachten die 1:1-Addukte 6a, 6b bzw. 20 und 21 und die 1:2-Addukte 18a, 18b, 19b bzw. 22 und 23. Das 1:1-Addukt 6b ging bei $120^{\circ} \mathrm{C}$, wahrscheinlich über die als Syntheseziel angestrebte Zwischenstufe $1 \mathrm{~b}$, in Benzol und die 1:2-Addukte $18 \mathrm{~b}, 19 \mathrm{~b}$ über, jedoch entstanden $18 \mathrm{~b}, 19 \mathrm{~b}$ in Gegenwart von Fumarsäure-dimethylester nicht mehr, und es bildete sich der $\Delta^{2}$-Isoxazolin-4,5-dicarbonsäure-dimethylester 24. - 1,4-Cyclohexadien nahm Benzo- und 2,4,6-Trimethylbenzonitriloxid zu den 1:1-Addukten 25 und den 1:2Addukten 26-28 auf. Die thermische Zersetzung von 25b lieferte Mesitylphenylketimin (31). Bei der Addition von Brom an 25a fiel ein 3:1-Gemisch der Dibromide 32 und 33 an. Die Behandlung von 32 mit Diazabicycloundecen ergab vermutlich das formale BenzolAddukt 12, jedoch wurden nur seine Folgeprodukte Benzol und Diphenylfuroxan (35) beobachtet. Unter der Einwirkung der stärkeren Base Kalium-tert-butoxid ging 32 in die Spiroverbindung 36 über.

Experiments to Synthesize a Formal Nitrile Oxide-Benzene Adduct

1,3-Dipolar Cycloadditions to Bicyclo[2.2.0]hex-5-ene-2,3-dicarboxylic Anhydride, Dewar Benzene, and 1,4-Cyclohexadiene

The reaction of bicyclo[2.2.0]hex-5-ene-2,3-dicarboxylic anhydride (7) with benzonitrile oxide, 2,4,6-trimethylbenzonitrile oxide, diphenylnitrile imine, diazomethane, tetrachloro-oquinone, and tetrachlorothiophene 1,1-dioxide furnished the $\Delta^{2}$-isoxazolines 8a, 8b, the $\Delta^{2}$ pyrazoline $8 \mathrm{c}$, the $\Delta^{1}$-pyrazoline $10 \mathrm{~d}$, the $\alpha$-diketone 11 , and the tetrachloro-1,3-cyclohexadiene derivative 12, respectively. - Dewar benzene (16) was prepared via the urazole 13, obtained from benzvalene by the addition of 4-methyl-4H-1,2,4-triazole-3,5-dione, and the azo compound 17 , from which nitrogen was eliminated by photolysis. The $1: 1$ adducts $6 \mathbf{a}$, $6 \mathrm{~b}, 20$, and 21 as well as the $1: 2$ adducts $18 \mathrm{a}, 18 \mathrm{~b}, 19 \mathrm{~b}, 22$, and 23 , respectively, were formed on treatment of 16 with benzonitrile oxide, 2,4,6-trimethylbenzonitrile oxide, and diazomethane. At $120^{\circ} \mathrm{C}$ the $1: 1$ adduct $6 \mathrm{~b}$ decomposed to give benzene and the $1: 2$ adducts $18 \mathrm{~b}, 19 \mathrm{~b}$, probably via the intermediate $1 \mathrm{~b}$, which was the target molecule of this investi-

(C) VCH Verlagsgesellschaft mbH, D-6940 Weinheim, 1985

$0009-2940 / 85 / 1010-4203 \$ 02.50 / 0$ 
gation. If the thermolysis was carried out in the presence of dimethyl fumarate, $18 \mathrm{~b}, \mathbf{1 9 b}$ were no longer observed and the dimethyl $\Delta^{2}$-isoxazoline-3,4-dicarboxylate 24 was obtained instead. - 1,4-Cyclohexadiene reacted with benzonitrile oxide and 2,4,6-trimethylbenzonitrile oxide to provide the $1: 1$ adducts 25 and the $1: 2$ adducts $26-28$. The thermal decomposition of $25 \mathrm{~b}$ resulted in the formation of mesitylphenylketimine (31). A 3:1 mixture of the dibromides 32 and 33 was obtained on addition of bromine to 25a. Elimination of hydrogen bromide from 32 was effected by diazabicycloundecene with benzene and diphenylfuroxan (35) being the products and the formal benzene adduct 1a the most likely intermediate. Compound 32 was transformed into the spiran 36 by using the stronger base potassium tert-butoxide.

Ziel dieser Arbeit war die Synthese eines formalen Benzol-Nitriloxid-Addukts 1, dessen Verhalten bei der Thermolyse interessierte. Würde 1 wegen des Gewinns an aromatischer Stabilisierungsenergie leicht der Cycloreversion zu Benzol und 1,3-Dipol anheimfallen oder könnte die Ringerweiterung zum Azoxonin 2 konkurrieren?

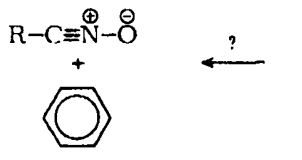

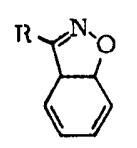

1

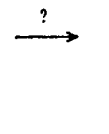<smiles>[R]c1ccccccon1</smiles>

2<smiles></smiles>

3

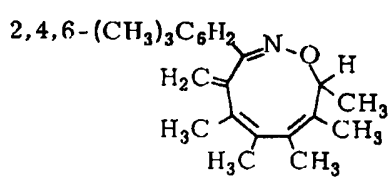

4

Immerhin bilden sich beim Erhitzen der Hexamethyldewarbenzoladdukte des Phenylazids und des 2,4,6-Trimethylbenzonitriloxids die neungliedrigen Monocyclen $3^{2)}$ bzw. $4^{3)}$.

\section{A. Cycloadditionen an Bicyclo[2.2.0]hex-5-en-2,3-dicarbonsäureanhydrid}

Benzol wird nur von den aggressivsten 1,3-Dipolen wie Ozon und Tetracyancarbonylylid ${ }^{4)}$, nicht aber von Nitriloxiden aufgenommen. Wir planten daher, 1 aus einem Valenzisomeren durch Umlagerung zu bereiten. Der leicht erfolgende Übergang zahlreicher Bicyclo[1.1.0]butane in 1,3-Butadienes) unter der Einwirkung von Silberionen ließ sich aber nicht auf das Benzonitriloxid-Addukt 5 des Benzvalens ${ }^{6}$ anwenden. Wahrscheinlich ist das Stickstoffatom ein so guter Komplexligand für $\mathrm{Ag}^{+}, \mathrm{da} B$ das gespannte $\sigma$-System bei niedriger Temperatur nicht konkurrieren konnte und unbehelligt blieb, während Erhitzen zu uneinheitlichem Zerfall führte. 
Als weiteres Valenzisomeres von 1a kam das Dewarbenzol-Addukt 6 in Frage, von dem zu erwarten war, da $\beta$ es bei $120^{\circ} \mathrm{C}$ die Cyclobuten-1,3-Butadien-Umlagerung zu $1 \mathrm{a}$ eingeht. Diese Annahme gründet sich auf die Bedingungen der Isomerisierung von Bicyclo[2.2.0]hex-2-en zu 1,3-Cyclohexadien ${ }^{\text {. }}$.

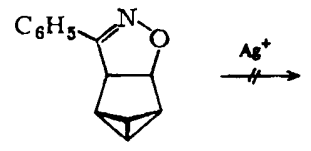

5

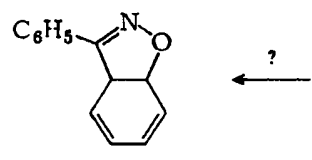

1a

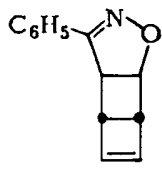

6

Dewarbenzol wird nach van Tamelen et al. ${ }^{8)}$ durch oxidative Bisdecarboxylierung von Bicyclo[2.2.0]hex-5-en-2,3-dicarbonsäureanhydrid (7) mit Bleitetraacetat oder von Bicyclo[2.2.0] hex-5-en-2,3-dicarbonsäure auf elektrochemischem Wege dargestellt. Beide Verfahren leiden unter bescheidenen Ausbeuten, weshalb wir versuchten, die Stufe des Dewarbenzols zu umgehen, d.h. die Cycloaddition bereits mit 7 und die oxidative Bisdecarboxylierung erst auf der Stufe eines Addukts auszuführen.
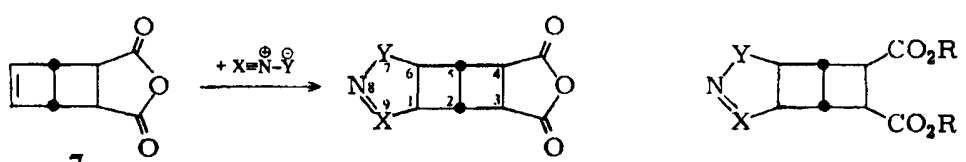

\begin{tabular}{l|ll|l|l} 
& $\mathrm{X}$ & $\mathrm{Y}$ & $\mathrm{R}=\mathrm{H}$ & $\mathrm{R}=\mathrm{CH}_{3}$ \\
\hline $\mathbf{8 a}$ & $\mathrm{C}_{6} \mathrm{H}_{5} \mathrm{C}$ & $\mathrm{O}$ & $\mathbf{9 a}$ & $\mathbf{1 0 a}$ \\
$\mathbf{8 b}$ & $2,4,6-\left(\mathrm{CH}_{3}\right)_{3} \mathrm{C}_{6} \mathrm{H}_{2} \mathrm{C}$ & $\mathrm{O}$ & $\mathbf{9 b}$ & $\mathbf{1 0 b}$ \\
$\mathbf{8 c}$ & $\mathrm{CC}_{6} \mathrm{H}_{5} \mathrm{C}$ & $\mathrm{NC}_{6} \mathrm{H}_{5}$ & $9 \mathbf{c}$ & $\mathbf{1 0 c}$ \\
& $\mathrm{N}$ & $\mathrm{CH}_{2}$ & & $10 d$
\end{tabular}

Wie erwartet reagierten die 1,3-Dipole Benzonitriloxid, 2,4,6-Trimethylbenzonitriloxid und Diphenylnitrilimin glatt mit 7 und lieferten in brauchbaren Ausbeuten die Anhydride 8a-c. Das lagerfähige 2,4,6-Trimethylbenzonitriloxid" wurde als solches zur Reaktion gebracht, während wir Benzonitriloxid ${ }^{10)}$ und Diphenylnitrilimin ${ }^{11)}$ jeweils in Gegenwart von 7 mit Triethylamin langsam aus Benzhydroximoylchlorid bzw. Benzphenylhydrazidchlorid freisetzten. Die Hydrolyse von 8a-c führte zu den Dicarbonsäuren 9a-c, und diese ließen sich wie üblich in die Dimethylester 10a - c umwandeln. Diazomethan in feuchtem Ether ergab mit 7 unmittelbar den Dimethylester 10d. Offenbar setzte Wasser schon bei $20^{\circ} \mathrm{C}$ die Säuregruppen aus der Anhydridfunktion frei, und erstere gingen mit

11

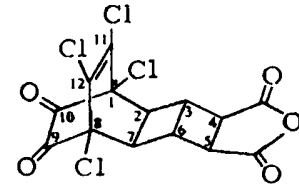

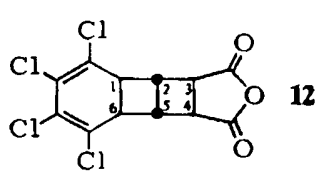

Chem. Ber. $118(1985)$ 
Diazomethan in Methylestergruppen über. Ob diese Reaktionsfolge vor oder nach der Cycloaddition an die CC-Doppelbindung abläuft, haben wir nicht untersucht.

Mit den reaktiven Dienen Tetrachlor-o-chinon ${ }^{12)}$ und Tetrachlorthiophen-1,1dioxid $^{13)}$ bildeten sich aus 7 die Diels-Alder-Produkte 11 bzw. 12.

Die Konfiguration der Verbindungen 8-12, d.h. die exo-Form des Ringgerüsts und die endo-Positionen der Carbonsäure-, -anhydrid- und -ester-Funktionen leiten wir aus dem Hochfeld-'H-NMR-Spektrum von $10 \mathrm{~d}$ ab, in dem durch Entkopplungsexperimente alle Parameter sicher zugeordnet werden konnten. Tab. 1 kennzeichnet $J_{1,2}, J_{2,3}, J_{4,5}$ und $J_{5,6}$ mit $2.5,2.5,3.0 \mathrm{bzw} .1 .0 \mathrm{~Hz}$ als trans-Kopplungen an ebenen Vierringen, $J_{1,6} J_{2,5}$ und $J_{3,4}$ mit $5.0,5.0 \mathrm{bzw} .9 .0 \mathrm{~Hz}$ aber als cis-Kopplungen. Die vergleichsweise kleinen Werte von $J_{1,6}$ und $J_{2,5}$ beruhen auf der elektronegativen Azofunktion an C-6 bzw. auf der Anellierung zweier Vierringe. Der letztere Effekt findet in Tricyclo[2.2.0.0 $\left.0^{2.6}\right]$ hexanen ${ }^{14)}$ eine Steigerung, wo die entsprechende Kopplungskonstante der synperiplanaren Protonen nur noch $1.0 \mathrm{~Hz}$ mißt.

Tab. 1. 'H-NMR-Chemische Verschiebungen ( $\delta$-Werte, Diagonalelemente) und Kopplungskonstanten (Absolutwerte in $\mathrm{Hz}$, Nichtdiagonalelemente) von 7,8-Diaza-exo-tricyclo[4.3.0.0 $0^{2.5}$ ]non-7-en-endo-3, endo-4-dicarbonsäure-dimethylester $(10 \mathrm{~d})$ in $\mathrm{CDCl}_{3}$. Leere Felder bedeuten $J<0.8 \mathrm{~Hz}$

\begin{tabular}{|c|c|c|c|c|c|c|c|c|}
\hline & $1-\mathrm{H}$ & $2-\mathrm{H}$ & 3-H & 4-H & $5-\mathrm{H}$ & $6-\mathrm{H}$ & $9-\mathrm{H}_{\text {endo }}$ & $9-\mathrm{H}_{\text {exo }}$ \\
\hline $\begin{array}{l}1-\mathrm{H} \\
2-\mathrm{H}\end{array}$ & 2.66 & $\begin{array}{l}2.5 \\
2.57\end{array}$ & $\begin{array}{l}2.5 \\
3.54\end{array}$ & 90 & 5.0 & 5.0 & 2.4 & 8.9 \\
\hline $\begin{array}{l}4-\mathrm{H} \\
5-\mathrm{H} \\
6-\mathrm{H} \\
9-\mathrm{H}_{\text {endo }} \\
9-\mathrm{H}_{\text {exo }}\end{array}$ & & ${ }_{10 d}^{\text {H }}$ & & 3.80 & $\begin{array}{l}3.0 \\
3.18\end{array}$ & $\begin{array}{l}1.0 \\
5.41\end{array}$ & $\begin{array}{l}2.4 \\
4.60\end{array}$ & $\begin{array}{c}1.5 \\
18.4 \\
4.70\end{array}$ \\
\hline
\end{tabular}

$\mathrm{OCH}_{3}: 3.69$ (s), 3.72 (s).

Das angestrebte Ziel, aus dem Anhydrid 8a oder der Dicarbonsäure 9a durch oxidative Bisdecarboxylierung das Dewarbenzol-Addukt 6 darzustellen, wurde nicht erreicht. Unter verschiedenen Bedingungen traten mit Bleitetraacetat in Pyridin ${ }^{15)}$ nicht näher untersuchte Ausweichprozesse ein.

\section{B. Cycloadditionen von Nitriloxiden und Diazomethan an Dewarbenzol}

Dieser Mißerfolg zwang uns, Dewarbenzol selbst herzustellen und zur Cycloaddition zu bringen. Die wenig ergiebigen Verfahren von van Tamelen et al. meidend, hofften wir darauf, daß sich die beschriebene Bildung von Dewarbenzol bei der Photolyse der Azoverbindung 17 ${ }^{16)}$ - "the yield of Dewar benzene is substantial" 16c) $-z u$ einer brauchbaren Synthese ausbauen ließe. 17 war erstmals von Trost et al. ${ }^{17)}$ dargestellt worden, ist aber ökonomischer nach Katz und Acton $^{16 \mathrm{~b})}$ ausgehend von Cyclopentadien in drei Stufen erhältlich. Letzterer Weg nutzt die Addition von 4-Phenyl-4H-1,2,4-triazol-3,5-dion an Benzvalen zu 15, gefolgt von der üblichen Umwandlung von Urazolen in Azoverbindungen. Versuchsbeschreibungen sind jedoch nicht veröffentlicht. 
Wegen der leichter eintretenden Alkoholyse von Methyl- im Vergleich zu Phenylurazolen, lagerten wir 4-Methyl-4H-1,2,4-triazol-3,5-dion an Benzvalen, wobei 13 praktisch quantitativ entstand und durch Chromatographie mit $55 \%$ Ausbeute in reiner Form isoliert wurde. Durch partielle Hydrolyse von 13 gelangten wir zu $63 \%$ 14; die vollständige Hydrolyse und die anschließende Oxidation erbrachte bezogen auf 13 mit $64 \%$ Ausbeute 17. Katz und Acton ${ }^{16 b}$ hatten die zu 13 analoge Verbindung 15 mit 50-60\% und daraus 17 mit $65 \%$ Ausbeute erhalten.

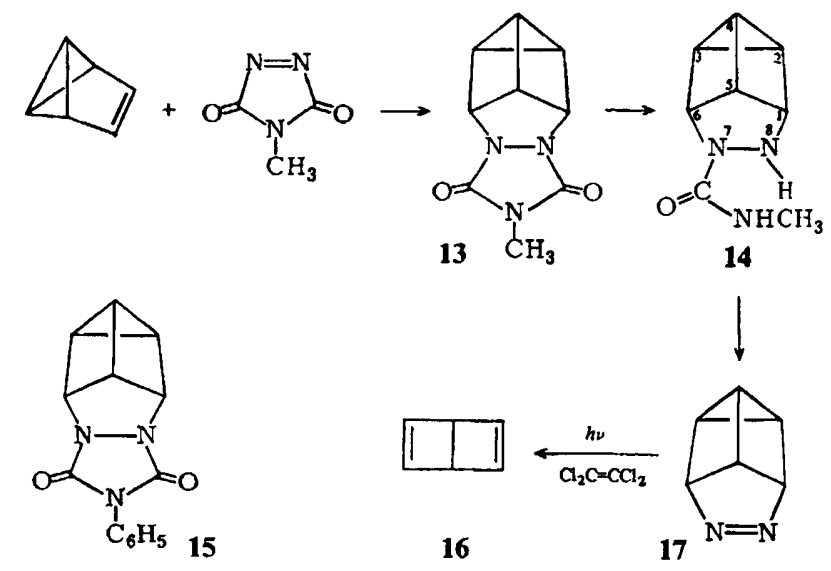

Nun kam es auf das Ergebnis der Photolyse von 17 an, bei der abhängig von den Bedingungen in verschiedenen Verhältnissen 1,8-Diazacyclooctatetraen, Benzol, Benzvalen, Prisman und Dewarbenzol (16) gefunden worden waren ${ }^{16}$. Wir versuchten, ein Lösungsmittel auszuwählen, in dem aufgrund publizierter Erkenntnisse ${ }^{160)}$ die Bildung von möglichst viel 16 zu erwarten war, das aber die bei der Photolyse entstehenden Polymeren möglichst gut löst und es gestattet, einigermaßen konzentrierte 16-Lösungen zu gewinnen. Tetrachlorethen erfüllte diesen Kompromiß am besten, wobei die Ausbeute an 16 maximal bei $11 \%$ lag und durch Umkondensieren bis zu 6proz. Lösungen anfielen. Wenn die Gesamtausbeute für die Umwandlung von Benzvalen in Dewarbenzol (16) auch höchstens $3.8 \%$ betrug, so konnten wir Mengen von ca. $300 \mathrm{mg}$ mit einem vertretbaren Aufwand in drei Stufen aus $8 \mathrm{~g}$ Benzvalen herstellen.

Bisher sind nur wenige Reaktionen des unsubstituierten Dewarbenzols (16) beschrieben, darunter eine 1,3-dipolare Cycloaddition, nämlich die des Diphenylnitrilimins ${ }^{8 c)}$. Man hatte ein Bisaddukt erhalten, aber weder die Stereochemie noch die Orientierung der Zweitaddition aufgeklärt.

Wir kamen bei der Reaktion von Benzonitriloxid mit $16 \mathrm{zu}$ einem Gemisch aus dem 1:1-Addukt 6a und dem 1:2-Addukt 18a mit Ausbeuten von 38 bzw. 21\%. Analog lieferte 2,4,6-Trimethylbenzonitriloxid $6 \mathrm{~b}$ und $18 \mathrm{~b}$, jedoch enthielt das Spektrum des Rohprodukts konkrete Hinweise auf das parallele Bisaddukt 19b mit dem Verhältnis 18b:19b $=7: 3$. Ein Gemisch dieser Komponenten im Verhältnis 2:1 bildete sich bei der Thermolyse von 6b (siehe Abschnitt C). Durch 
Chromatographie, Destillation und Kristallisation wurden die Verbindungen 6 und 18 in reiner Form isoliert. Aus der Umsetzung von Diazomethan mit 16 gingen die vier Produkte 20-23 hervor. Von den Bisaddukten 22 und 23 fiel ein reines 55:45-Gemisch mit 32\% Ausbeute an, während das zersetzliche 10:1-Gemisch der 1:1-Addukte 20 und 21 (30\% Ausbeute) noch 22 und 23 enthielt.

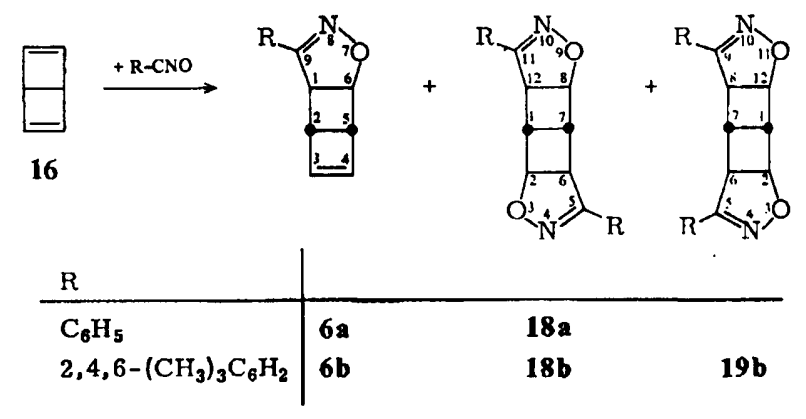

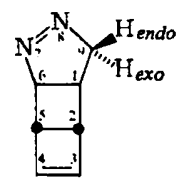

20

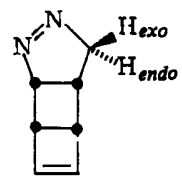

21

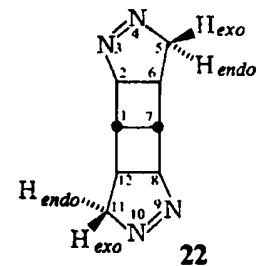

22

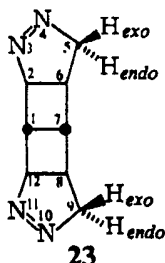

23

Die Strukturen der Verbindungen 6 und 18-23 und ihre Konfiguration folgen aus den NMR-Spektren. So wird die exo-Anellierung der Ringe in 6 und 20 durch die Werte der Kopplungskonstanten $J_{1,2}$ und $J_{5,6}$ von ca. $1 \mathrm{~Hz}$ angezeigt, die nur mit einer trans-Anordnung der betreffenden Protonen am ebenen Vierring vereinbar sind.

Der Tab. 2 entnimmt man anhand der Parameter von 6b - sehr ähnliche Werte weisen 6a und 20 auf - , daB in exo-anellierten Bicyclo[2.2.0]hexen-Derivaten die kleinen Kopplungskonstanten vorherrschen. Selbst die cis-Kopplung $J_{2,5}$ miBt nur $2.3 \mathrm{~Hz}$, und lediglich $J_{1,6}=5.7 \mathrm{~Hz}$ fällt aus dem Rahmen, ist aber auch kleiner als die entsprechende cisKopplung in gewöhnlichen $\Delta^{2}$-Isoxazolinen ${ }^{18)}$. Da Entkopplungsexperimente nicht ausgeführt wurden, nahmen wir zur Zuordnung der 5-H- und 4-H-Signale an, daB sie wegen der größeren Nähe dieser Protonen zum elektronegativen Sauerstoffatom verglichen mit 2-H bzw. 3-H bei tieferem Feld erscheinen als die 2-H- bzw. 3-H-Resonanzen. Diese Regel setzten wir auch bei der Zuweisung der 1-H- und 6-H-Absorptionen voraus ${ }^{18}$.

Wie Tab. 3 zeigt, verfügt das Addukt 21, dessen 3- und 4-H-Signale eine Feinstruktur gleich der der entsprechenden Resonanzen von 20 aufweisen, über große $J_{1,2^{-}}$und $J_{5,6}$-Werte $(7.0$ und $8.0 \mathrm{~Hz}$ ), was nur mit der cis-Anordnung dieser Protonen am ebenen Vierring und so mit der endo-Anellierung der Ringe in 21 im Einklang steht. Die Zuordnung innerhalb der von 2-, 5-H und 3-, 4-H herrührenden Signalpaare wurde in Analogie zu den Verbindungen 6 so getroffen, daß jeweils das Tieffeld-Signal vom Proton herrührt, das N-7 näher ist.

Die Orientierung des zweiten Heterocyclus bei den 1:2-Addukten läßt sich aus der Zahl der Signale in den 'H- und ${ }^{13} \mathrm{C}-\mathrm{NMR}$-Spektren ablesen. Weil das Bicyclo[2.2.0]hexan-Sy- 
stem nur drei intensitätsgleiche Signale verursacht, müssen 18 und $22 c_{2}$-Symmetrie besitzen. Dagegen folgt die $c_{3}$-Symmetrie von 23 aus vier Absorptionen im Intensitätsverhältnis 2:2:1:1. Zwar weisen die zu 18, 22 und 23 gehörigen endo,endo-Diastereomeren die gleiche Symmetrie auf wie die ersteren, jedoch sprechen die kleinen Kopplungskonstanten $J_{1,2}$ und $J_{6,7}$ gegen diese Alternativen.

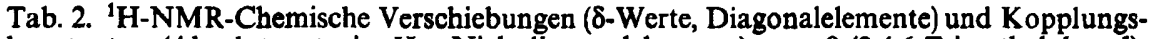
konstanten (Absolutwerte in $\mathrm{Hz}$, Nichtdiagonalelemente) von 9-(2,4,6-Trimethylphenyl)7-oxa-8-aza-exo-tricyclo[4.3.0.0 $0^{2.5}$ ]nona-3,8-dien (6b) in $\mathrm{CDCl}_{3}$. Leere Felder bedeuten $J<0.3 \mathrm{~Hz}$

\begin{tabular}{lllllll}
\hline & $1-\mathrm{H}$ & $2-\mathrm{H}$ & $3-\mathrm{H}$ & $4-\mathrm{H}$ & $5-\mathrm{H}$ & $6-\mathrm{H}$ \\
\hline $1-\mathrm{H}$ & 4.10 & 0.9 & & & & 5.7 \\
$2-\mathrm{H}$ & & 3.52 & 2.2 & 0.5 & 2.3 & 0.5 \\
$3-\mathrm{H}$ & & 6.22 & 2.4 & 0.6 & \\
$4-\mathrm{H}$ & & & 6.29 & 2.4 & \\
$5-\mathrm{H}$ & & & & 3.77 & 0.6 \\
$6-\mathrm{H}$ & & & & & 5.09 \\
\hline
\end{tabular}

$\mathrm{R}=2,4,6-\left(\mathrm{CH}_{3}\right)_{3} \mathrm{C}_{6} \mathrm{H}_{2}: 2.38\left(p-\mathrm{CH}_{3}\right), 2.39\left(o-\mathrm{CH}_{3}\right), 6.91(m-\mathrm{H})$ (jeweils br. s).

Tab. 3. 'H-NMR-Chemische Verschiebungen ( $\delta$-Werte, Diagonalelemente) und Kopplungskonstanten (Absolutwerte in $\mathrm{Hz}$, Nichtdiagonalelemente) von 7,8-Diaza-endo-tricyclo[4.3.0.0 $0^{2.5}$ nona-3,7-dien (21) in $\mathrm{C}_{6} \mathrm{D}_{6}$. Leere Felder bedeuten $J<0.5 \mathrm{~Hz}$

\begin{tabular}{|c|c|c|c|c|c|c|c|c|}
\hline & $1-H$ & $2-\mathrm{H}$ & $3-\mathrm{H}$ & $4-\mathrm{H}$ & $5-H$ & $6-\mathrm{H}$ & 9- $\mathrm{H}_{\text {endo }}$ & $9-\mathrm{H}_{\text {exo }}$ \\
\hline $\begin{array}{l}1-\mathrm{H} \\
2-\mathrm{H} \\
3-\mathrm{H} \\
4-\mathrm{H} \\
5-\mathrm{H} \\
6-\mathrm{H} \\
9-\mathrm{H}_{\text {endo }} \\
9-\mathrm{H}_{\text {exo }}\end{array}$ & 1.87 & $\begin{array}{l}7.0 \\
2.63 \\
\\
4\end{array}$ & $\begin{array}{l}2.3 \\
5.63\end{array}$ & $\begin{array}{l}0.6 \\
2.3 \\
5.82\end{array}$ & $\begin{array}{l}2.5 \\
0.6 \\
2.3 \\
3.26\end{array}$ & $\begin{array}{l}8.0 \\
5.00\end{array}$ & $\begin{array}{l}3.2 \\
3.80\end{array}$ & $\begin{array}{c}1.7 \\
18.0 \\
3.73\end{array}$ \\
\hline
\end{tabular}

Alle Versuche belegen den starken Vorzug der 1,3-dipolaren Cycloadditionen an Dewarbenzol (16) von der exo-Seite her. Möglicherweise ist das bei 20 und 21 ermittelte Verhältnis exo:endo $\approx 10: 1$ repräsentativ. Bei den Nitriloxid-Additionen könnten derartig kleine Anteile an endo-Isomeren der Untersuchung entgangen sein. Da nur exo,exo-1:2-Produkte gefunden wurden, ist der endo-Angriff bei der Zweitaddition eher noch stärker benachteiligt. Dies muß man wegen der engeren räumlichen Verhältnisse aufgrund der endo-ständigen 1-, 6-H-Atome am exo-anellierten Bicyclo[2.2.0]hexen-System in 6 und 20 auch erwarten. Bei den Nitriloxid-Reaktionen isolierten wir nur die antiparallelen 1:2-Addukte 18, erkannten aber eine erhebliche Menge des parallelen Addukts 19 im Rohprodukt. Im Falle der Diazomethan-Anlagerung traten die Produkte beider Angriffsrichtungen nahezu gleichberechtigt auf. Offensichtlich übt der erste Heterocyclus nur einen geringfügigen dirigierenden Einfluß auf die Orientierung der Zweitaddition aus.

Chem. Ber. 118 (1985) 


\section{Die Thermolyse des Dewarbenzol-Addukts 6b}

Durch die Thermolyse von $6 \mathrm{~b}$ hofften wir, zum formalen Benzol-Addukt $1 \mathrm{~b}$ zu kommen. Aufgrund der Zerfallstemperatur von Bicyclo[2.2.0] hex-2-en ${ }^{7}$ erhitzten wir auf $120^{\circ} \mathrm{C}$. Laut NMR-Analyse ging dabei $6 \mathrm{~b}$ innerhalb von acht Stunden vollständig in Benzol und die 1:2-Addukte 18b und 19b über, letztere im Verhältnis 2:1. Wahrscheinlich war 1b entstanden, aber sofort in Benzol und Nitriloxid zerfallen, das sich an die CC-Doppelbindung von noch vorhandenem $6 \mathrm{~b}$ lagerte.

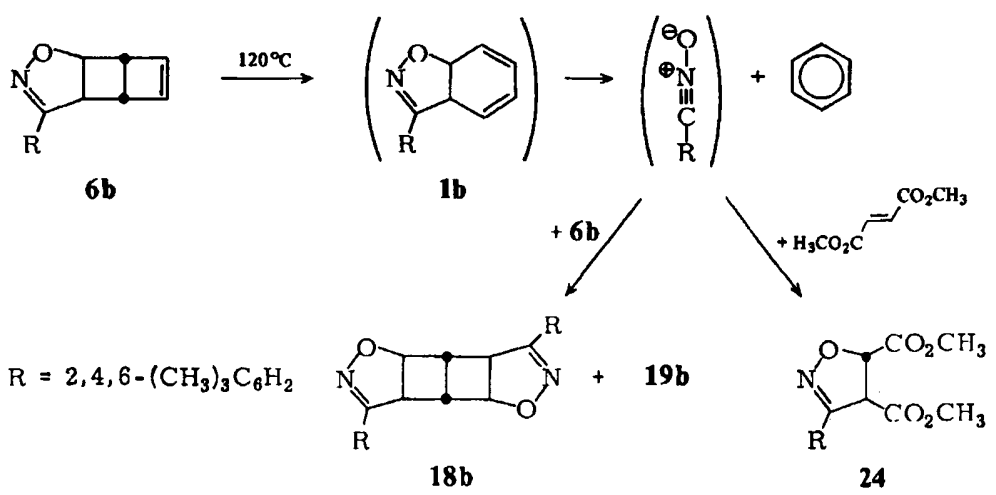

Um das vermutete freie Auftreten von 2,4,6-Trimethylbenzonitriloxid nachzuweisen, thermolysierten wir 6b in Gegenwart von Fumarsäure-dimethylester, den eine hohe Reaktivität gegenüber Benzonitriloxid auszeichnet ${ }^{19,20)}$ und der daher 6b in der Konkurrenz um das Nitriloxid den Rang ablaufen sollte. Tatsächlich bildete sich jetzt neben Benzol nur der Isoxazolindicarbonester 24, der durch Vergleich mit einem unabhängig dargestellten Präparat identifiziert wurde.

Offenbar kann durch die thermische Cyclobuten-1,3-Butadien-Isomerisierung aus $6 \mathrm{~b}$ bei $120^{\circ} \mathrm{C}$ das formale Benzol-Addukt $1 \mathrm{~b}$ generiert werden, jedoch erleidet es, ohne beobachtet worden zu sein, sehr rasch die 1,3-dipolare Cycloreversion, besonders gefördert durch die aromatische Stabilisierungsenergie des Spaltprodukts Benzol.

\section{Der Weg über das 1,4-Cyclohexadien-Addukt 25a}

Wegen des raschen Zerfalls von $1 \mathrm{~b}$ bei $120^{\circ} \mathrm{C}$ bemühten wir uns um einen Zugang zu 1 bei niedriger Temperatur. Eine Route, auf der man recht allgemein von einem Cyclohexenzu einem 1,3-Cyclohexadien-Derivat gelangt, besteht in der Addition von Brom gefolgt von der zweifachen Bromwasserstoff-Eliminierung. Ausgangsmaterial für 1 wäre somit ein 1:1Addukt 25 eines Nitriloxids an 1,4-Cyclohexadien. Verbindungen vom Typ 25 finden sich bisher nicht in der Literatur ${ }^{19}$.

Es stellte sich heraus, daß 1,4-Cyclohexadien gegenüber Benzonitriloxid und Trimethylbenzonitriloxid nicht sehr reaktionsfreudig ist. Mit guter Ausbeute erhielten wir das 1:1-Addukt 25a nur bei Anwendung eines großen Uberschusses an 1,4-Cyclohexadien in hoher Konzentration. Wurden dagegen Dipolarophil und

Chem. Ber. 118 (1985) 
1,3-Dipol im Verhältnis 2:1 zur Reaktion gebracht, so ergaben sich 1:2-Addukte als Hauptprodukte und nur bescheidene Ausbeuten an 25a und 25b.

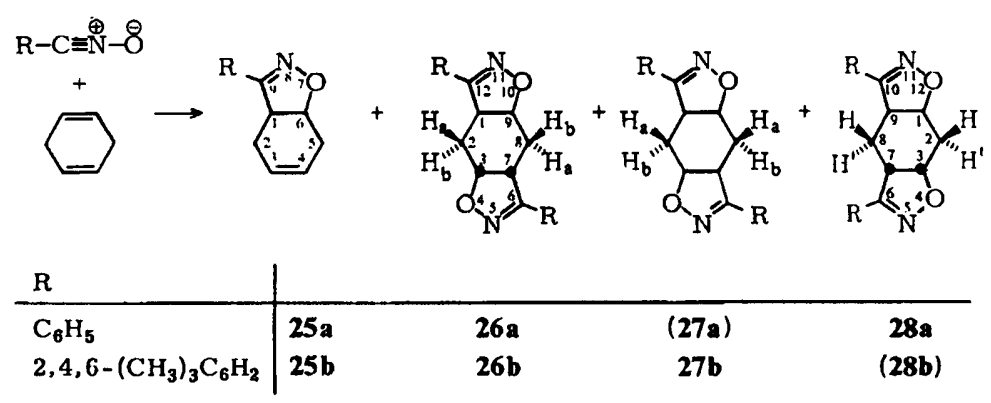

Unter der Voraussetzung, daß die 1,3-dipolare Cycloaddition vollständig cis-stereoselektiv verläuft, sind zwei parallele und zwei antiparallele Bisaddukte möglich, die durch ihre NMRParameter identifizierbar sein sollten. Im Benzonitriloxid-Fall fanden wir das antiparallele 26a und das parallele 28a im Verhältnis 1.4:1. Die Cyclohexan-Einheit von 26a ist durch drei ${ }^{13} \mathrm{C}$-NMR-Signale gleicher Intensität, jene von $\mathbf{2 8 a}$ aber durch vier mit dem Intensitätsverhältnis 2:2:1:1 charakterisiert. Daß die exo-Verbindung 28a und nicht ihr endoIsomeres mit beiden Heterocyclen auf einer Seite des Sechsrings vorlag, folgt aus dem ${ }^{1} \mathrm{H}$ NMR-Spektrum, das die geminalen Kopplungen in den $\mathrm{CH}_{2}$-Gruppen nicht unmittelbar preisgibt, weil es sich bei den Signalen um AA' $^{\prime}$-Teile von AA' $^{\prime} \mathbf{M M}^{\prime}$-Spektren mit weiter zum Dublett aufgespaltenen $M M^{\prime}$-Teilen handelt. Dagegen sind für das Diastereomere von 28a zwei $\mathrm{ABX}_{2}$-Spektren mit weiter zum Dublett aufgespaltenen $\mathrm{X}_{2}$-Teilen zu erwarten, welche die geminalen Kopplungen der $\mathrm{CH}_{2}$-Gruppen $\left(J_{A, B}\right)$ direkt meßbar enthalten.

Die Entscheidung, ob 26a oder 27 a angefallen war, konnte nur durch Vergleich mit den ${ }^{1} \mathrm{H}$-NMR-Kopplungskonstanten von $26 \mathrm{~b}$ und $27 \mathrm{~b}$ getroffen werden, zu deren 6.5:1-Gemisch wir im Trimethylbenzonitriloxid-Fall gelangten. Aufgrund der Molekülmodelle dürfte in diesen Verbindungen der Sechsring eine Bootkonformation annehmen, wobei eine Verdrillung in Richtung Twistform an folgenden Argumenten nichts ändert. Die Inversion der Bootform 26 des exo-Isomeren, die sehr rasch verlaufen dürfte, führt zur energiegleichen Bootform 26'. Die Interplanarwinkel der Protonenpaare 1-H/2- $\mathrm{H}_{\mathrm{a}}$ und $2-\mathrm{H}_{\mathrm{b}} / 3-\mathrm{H}$ betragen also etwa $180 \mathrm{bzw} .60^{\circ}$ in 26 und 60 bzw. $180^{\circ}$ in $26^{\circ}$, womit die gefundenen Kopplungskonstanten im Rahmen der Karplus-Conroy-Beziehung gut übereinstimmen: 26a: $J_{1,2 \mathrm{a}}=$ $7.5, J_{2 \mathrm{~b}, 3}=7.2 \mathrm{~Hz} ; 26 \mathrm{~b}: J_{1.2 \mathrm{a}}=7.0, J_{2 \mathrm{~b}, 3}=7.4 \mathrm{~Hz}$. Für die Protonenpaare $1-\mathrm{H} / 2-\mathrm{H}_{\mathrm{b}}$ und 2- $\mathrm{H}_{\mathrm{a}} / 3-\mathrm{H}$ belaufen sich aber die Interplanarwinkel in 26 und $26^{\circ}$ auf $60^{\circ}$ und erklären so die kleineren Kopplungskonstanten: $26 \mathrm{a}: J_{1,2 \mathrm{~b}}=5.7, J_{2 \mathrm{a}, 3}=4.4 \mathrm{~Hz} ; 26 \mathrm{~b}: J_{1,2 \mathrm{~b}}=6.9, J_{2 \mathrm{a}, 3}=$ $4.3 \mathrm{~Hz}$. Wie bei $\Delta^{2}$-Isoxazolinen üblich ${ }^{18)}$, gewährleistet die Differenz der chemischen Verschiebungen der beiden Protonen am Heterocyclus die zweifelsfreie Zuordnung.

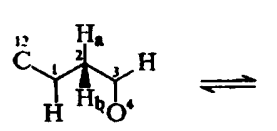

26<smiles>C=CCC(C)C(C)O</smiles>

$26^{\prime}$<smiles>C=CC(C)C(C)C(C)OC</smiles>

27<smiles>C=CC(=O)[C@H](O)C(C)C</smiles>

$27^{\prime}$

Das zweite Bisaddukt im Trimethylbenzonitriloxid-Fall muBte aufgrund der Zahl seiner ${ }^{13} \mathrm{C}-\mathrm{NMR}$-Signale ebenfalls von antiparalleler Natur sein, und die Werte der vicinalen Kopp-

Chem. Ber. 118 (1985) 
lungskonstanten von 2- $\mathrm{H}_{\mathrm{a}}(13.6$ und $11.1 \mathrm{~Hz})$ und $2-\mathrm{H}_{\mathrm{b}}(5.6$ und $6.0 \mathrm{~Hz})$ sprachen für das endo-Isomere 27b. Die Formeln 27 und 27' repräsentieren die beiden, durch Inversion ineinander übergehenden Bootkonformationen, von denen aber $27^{\prime}$ wegen der 1,3-diaxialen Positionen von $\mathrm{C}-12$ und $\mathrm{O}-4$ energetisch stark benachteiligt und daher kaum populiert sein dürfte. In 27 weist ein Proton der $\mathrm{CH}_{2}$-Gruppe $\left(\mathrm{H}_{\mathbf{2}}\right)$ zwei antiperiplanare Kopplungspartner, das andere $\left(\mathrm{H}_{\mathrm{b}}\right)$ aber zwei synclinale auf, was mit dem großen Unterschied zwischen den zwei Typen von Kopplungskonstanten im Einklang steht.

Bemerkenswert ist, daß wir aus 1,4-Cyclohexadien und Benzonitriloxid neben 26a relativ viel des parallelen Bisaddukts 28 a erhielten, während das analoge 28 b beim Einsatz von Trimethylbenzonitriloxid nicht auftauchte. Möglicherweise äußert sich hier der größere Raumanspruch der Mesitylgruppen, der trotz ihres großen Abstandes den Úbergangszustand auf dem Weg zu $28 \mathrm{~b}$ energiereicher macht. Allerdings untersuchten wir nur die anfallenden Kristalle, so daB sich die vielleicht in den Rohprodukten vorhandenen 27 a bzw. $28 \mathrm{~b}$ und auch die Stereoisomeren zu $28 \mathrm{a}$ und $28 \mathrm{~b}$ der Identifizierung entzogen haben könnten.

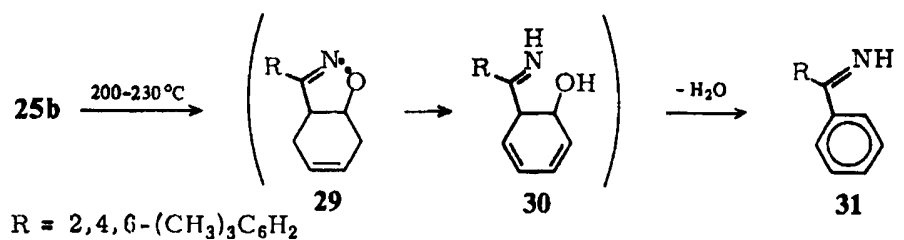

Beim Erhitzen von $25 \mathrm{~b}$ auf $200-230^{\circ} \mathrm{C}$ bildete sich das bekannte ${ }^{21)}$ Imin 31. Wahrscheinlich beginnt dieser Zerfall mit dem Bruch der schwāchsten Bindung zum Diradikal 29, das durch zweifache H-Atom-Übertragung in 30 übergehen könnte, und daraus resultierte durch Eliminierung von Wasser 31.

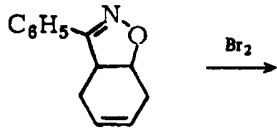

25a

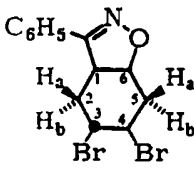

32

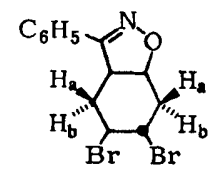

33

Die Addition von Brom an 25a führte mit hoher Ausbeute zu zwei Diastereomeren im Verhältnis 3:1. Entkopplungsexperimente im ${ }^{1} \mathbf{H}-\mathrm{NMR}$-Spektrum des Überschußisomeren gewährleisteten die eindeutige Zuordnung aller Signale. Trotz einiger Signalüberlagerungen im Spektrum des Gemisches gelang auch eine konsistente Interpretation der Signale des Unterschußisomeren. Der wichtigste Unterschied in den Parametern der beiden Produkte besteht in der Größenordnung der Kopplungskonstanten von 3- und 4-H. Während beim Überschußisomeren $J_{2 \mathrm{a}, 3}, J_{2 \mathrm{~b}, 3}, J_{3,4}, J_{4,5 \mathrm{z}}$ und $J_{4,5 \mathrm{~b}}$ zwischen 2.5 und $5.0 \mathrm{~Hz}$ messen, belaufen sich $J_{3,4}$ und jeweils einer der beiden $J_{2,5}$ und $J_{4,5}$-Werte des Unterschußisomeren auf 9.5 bzw. 11.0 und $11.5 \mathrm{~Hz}$. Unter der Voraussetzung, daß die übliche trans-Addition der beiden Bromatome stattgefunden hat, sind diese Daten am besten mit der Formel 32 für das UUberschußisomere und 33 für das Unterschußisomere vereinbar. Dabei müßte der Cyclohexanring jeweils die Sesselkonformation annehmen mit 
dem Isoxazolin-Sauerstoffatom, das im Vergleich mit dem Phenyl-tragenden CAtom erheblich weniger Raum beansprucht, in einer axialen Position, womit die Bromatome in 32 axiale, in 33 aber äquatoriale Stellungen zu beziehen hätten. Daraus ergäben sich Interplanarwinkel, die perfekt zu den ermittelten Kopplungskonstanten paßten.

Diese Konformation des bicyclischen Gerüsts hat einen von $0^{\circ}$ abweichenden Interplanarwinkel von 1-H und 6-H zur Folge. Ubereinstimmend damit fallen $J_{1,6}$ mit 6.9 (32) und $6.8 \mathrm{~Hz}$ (33) kleiner aus als bei vielen anderen $\Delta^{2}$-Isoxazolinen ${ }^{18)}$ (vgl. auch 25-28 mit 9.7-11.4 Hz für die entsprechenden Kopplungen). Darüber hinaus liegen alle ${ }^{13} \mathrm{C}-\mathrm{NMR}$ Absorptionen von 32 bei zum Teil erheblich höherem Feld als die von 33, was auf stärkere Gruppenhäufung hindeutet, wohl hervorgerufen durch die axial angeordneten Bromatome.

Zur Umwandlung von 32 in 1a bedurfte es der Eliminierung von zwei Molekülen Bromwasserstoff, was wir mit der bewährten Base Diazabicycloundecen (DBU) versuchten. Tatsächlich schieden Lösungen der Reaktionspartner in $\mathrm{C}_{6} \mathrm{D}_{6}$ oder Tetrahydrofuran bei $20^{\circ} \mathrm{C}$ innerhalb von $24 \mathrm{~h}$ eine große Menge eines Niederschlags ab, vermutlich DBU $\cdot \mathrm{HBr}$, und die ${ }^{1} \mathrm{H}$-NMR-Signale von 32 verloren stark an Intensität, aber es entwickelten sich nur schwache Signale im Absorptionsbereich olefinischer Protonen, die möglicherweise 1a oder seiner unmittelbaren Vorstufe nach Eliminierung von einem Molekül $\mathrm{HBr}$ aus 32 zugeschrieben werden können. Ein Singulett bei $\delta=7.2$ sprach für die Bildung von Benzol, und auch die Isolierung von Diphenylfuroxan (35) aus dem Reaktionsgemisch legte nahe, da $B$ das angestrebte $1 \mathrm{a}$ mit hoher Wahrscheinlichkeit entstanden, aber selbst bei $20^{\circ} \mathrm{C}$ rasch der 1,3-dipolaren Cycloreversion unterlegen war, der sich die Dimerisierung des Benzonitriloxids ${ }^{19)} \mathrm{zu} 35$ anschloB.

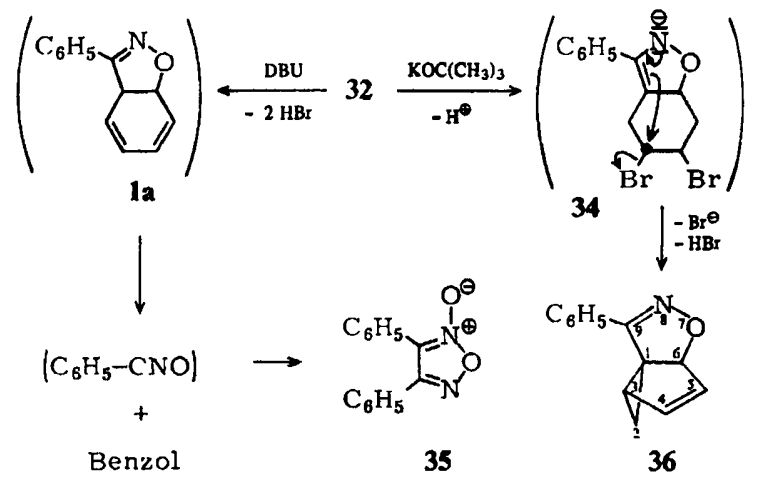

Der Einsatz der stärkeren Base Kalium-tert-butoxid zwecks Beschleunigung der HBr-Abspaltung änderte den Reaktionsablauf dramatisch, denn es bildete sich die Spiroverbindung 36. Offenbar gewinnt statt der erwünschten E2-Eliminierung ein E1-Ablauf die Oberhand, weil jetzt die Base das Proton der 1-Stellung, das acideste Proton von 32 übernimmt. Im Enamid-Ion 34 tritt dann eine intramolekulare Alkylierung unter Abschnürung des dreigliedrigen Rings ein, und schließlich muß ein E2-Schritt 36 hervorbringen. Bei 5-(Brom- oder Chlormethyl)-3-phenyl- $\Delta^{2}$ -

Chem. Ber. 118 (1985) 
isoxazolin ist durch Umsetzung mit Basen ein ähnlicher Ringschluß erzielt worden ${ }^{22}$.

Tab. 4. 'H-NMR-Chemische Verschiebungen ( $\delta$-Werte, Diagonalelemente) und Kopplungskonstanten (Absolutwerte in $\mathrm{Hz}$, Nichtdiagonalelemente) von 9-Phenyl-7-oxa-8-azatricyclo[4.3.0.0 ${ }^{1,3}$ ]nona-4,8-dien (36) in $\mathrm{CDCl}_{3}$. Leere Felder bedeuten $J<0.5 \mathrm{~Hz}$

\begin{tabular}{lllllll}
\hline & $2-\mathrm{H}_{\text {exo }}$ & $2-\mathrm{H}_{\text {endo }}$ & $3-\mathrm{H}$ & $4-\mathrm{H}$ & $5-\mathrm{H}$ & $6-\mathrm{H}$ \\
\hline $2-\mathrm{H}_{\text {exo }}$ & 2.14 & 5.6 & 8.0 & & 0.5 & 0.5 \\
$2-\mathrm{H}_{\text {endo }}$ & & 0.95 & 4.6 & & & 2.7 \\
$3-\mathrm{H}$ & & & 2.42 & 1.8 & 5.5 & 1.2 \\
$5-\mathrm{H}$ & & & 6.32 & 5.97 & 1.7 \\
$6-\mathrm{H}$ & & & & & 5.49 \\
& & & & & & \\
\hline
\end{tabular}

$\mathrm{C}_{6} \mathrm{H}_{5}: 7.38-7.43(3 \mathrm{H}), 7.46-7.50(2 \mathrm{H})$.

Tab. 4 enthält die 'H-NMR-Parameter von 36, deren Zuordnung durch Entkoppeln gestützt wurde. Problematisch war die Unterscheidung der Signale der Olefinprotonen 4 und 5-H. Aufgrund der Erfahrung mit ähnlichen Systemen ${ }^{23 a}$ gab das Argument den Ausschlag, daß die vicinale Kopplung $J_{3,4}$ in jedem Fall meßbar groß sein sollte, während die Allylkopplung $J_{3,5}$ durchaus die Auflösungsgrenze unterschreiten könnte. Beachtung verdienen die Fernkopplungen von $2-\mathrm{H}_{\text {exo }} \mathrm{zu} 5-\mathrm{H}$ und 6-H, und die Fernkopplung $J_{3,6}=2.7 \mathrm{~Hz}$ stützt die trans-Anordnung dieser Protonen am Cyclopentenring ${ }^{236)}$ und so die cis-Anellierung des Isoxazolinrings.

Unser Dank gilt dem Fonds der Chemischen Industrie für die Förderung der Arbeit, sowie der Union Rheinische Braunkohlen Kraftstoff $A G$ für Dimethylether-Spenden. Herrn Dr. $H$. Leininger danken wir für erste Versuche zur Darstellung von 6b aus Benzvalen und Herrn Dr. D. Wendisch, Bayer AG, für die Aufnahme der $90.56-\mathrm{MHz}-{ }^{13} \mathrm{C}$ - und $360-\mathrm{MHz}-{ }^{1} \mathrm{H}-\mathrm{NMR}-$ Spektren von $10 \mathrm{~d}$ und die sorgfältigen Entkopplungsexperimente in den letzteren.

\section{Experimenteller Teil}

${ }^{1}$ H-NMR: Varian A 60 und EM 390, Bruker WH 360 und WM 400. - ${ }^{13} \mathrm{C}-\mathrm{NMR}$ : Bruker WH 90, WH 360 und WM 400. - MS: Varian MAT CH 7. - IR: Beckman AccuLab 4. - UV: Beckman DB-GT. - Schmelzpunkte (nicht korrigiert): Mikroheiztisch der Firma Reichert, Wien. - Elementaranalysen: Carlo Erba Strumentatione Elemental Analyzer Mod. 1106.

\section{A. Cycloadditionen an Bicyclo[2.2.0]hex-5-en-2,3-dicarbonsäureanhydrid (7)}

9-Phenyl-7-oxa-8-aza-exo-tricyclo [4.3.0.0.5 ]non-8-en-endo-3,endo-4-dicarbonsäureanhydrid (8a): Zur Lösung von $1.40 \mathrm{~g}(9.32 \mathrm{mmol}) 7^{8 \mathrm{~b}, 24)}$ und $1.45 \mathrm{~g}(9.32 \mathrm{mmol})$ Benzhydroximoylchlorid ${ }^{25)}$ in $30 \mathrm{ml}$ Ether und $30 \mathrm{ml}$ Dichlormethan tropfte man unter Kühlung mit Eis und Rühren innerhalb von $90 \mathrm{~min} 5.06 \mathrm{~g}$ (50.0 mmol) Triethylamin in $10 \mathrm{ml}$ Ether. Man rührte das Gemisch weitere $5 \mathrm{~h}$ im Eisbad, filtrierte den Niederschlag ab, digerierte ihn mit Wasser, filtrierte erneut ab und wusch die Kristalle mehrmals mit kaltem Ether. Das trockene Produkt wog $1.31 \mathrm{~g}$ und erbrachte beim Umlösen aus Aceton/Petrolether $\left(30-50^{\circ} \mathrm{C}\right) 1.15 \mathrm{~g}$ 
(46\%) $8 \mathrm{a}$ in farblosen Kristallen mit Schmp. $228-230^{\circ} \mathrm{C}$. - IR (KBr): $1860,1780 \mathrm{~cm}^{-1}$ $(\mathrm{C}=\mathrm{O}) .-\mathrm{MS}(70 \mathrm{eV}): \mathrm{m} / \mathrm{e}=269\left(13 \%, \mathrm{M}^{+}\right), 145(100) .-{ }^{\mathrm{J}} \mathrm{H}-\mathrm{NMR}\left[\left(\mathrm{CD}_{3}\right)_{2} \mathrm{CO}\right]: \delta=$ 3.28 (br. s; 2-, 5-H), 3.85 und 4.09 (br. AB-Spektrum, $J_{3,4}=5.3 \mathrm{~Hz}$; 3-, 4-H), 4.87 (br. d, $\left.J_{1,6}=6.0 \mathrm{~Hz} ; 1-\mathrm{H}\right), 5.45$ (br. d; 6-H), $7.2-7.8\left(\mathrm{~m} ; \mathrm{C}_{6} \mathrm{H}_{5}\right)$.

Dicarbonsäure 9a: $600 \mathrm{mg}(2.23 \mathrm{mmol}) 8 \mathrm{a}$ wurden in $10 \mathrm{ml}$ Tetrahydrofuran und $1 \mathrm{ml}$ Wasser 6 h unter Rückfluß gekocht. Nach dem Abkühlen verdünnte man mit Wasser und verdampfte das Tetrahydrofuran i.Vak., wobei $135 \mathrm{mg} \mathrm{(21 \% )} \mathrm{9a} \mathrm{als} \mathrm{farblose} \mathrm{Würfel} \mathrm{ausfie-}$ len, die bei $155-160^{\circ} \mathrm{C}$ zu schmelzen begannen und sich dann in lange dünne Nadeln mit Schmp. $227-230^{\circ} \mathrm{C}$ umwandelten. Vermutlich trat bei diesem Vorgang die Rückbildung des Anhydrids 8a ein. - IR (KBr): 3700-2300 (breit, $\mathrm{O}-\mathrm{H}$ ), 1715, $1658 \mathrm{~cm}^{-1}(\mathrm{C}=\mathrm{O})$. MS (70 eV): Übereinstimmend mit MS von 8a. $-{ }^{1} \mathrm{H}-\mathrm{NMR}\left[\left(\mathrm{CD}_{3}\right)_{2} \mathrm{CO}\right]: \delta=3.17$ (br. s; 2-, 5-H), 3.69 und 3.88 (jeweils dd, $J_{3,4}=8.4, J_{2,3}=J_{4,5}=2.2 \mathrm{~Hz} ; 3-, 4-\mathrm{H}$ ), 4.58 (br. d, $J_{1,6}=$ $5.9 \mathrm{~Hz} ; 1-\mathrm{H}$ ), 5.28 (br. d; 6-H), 5.85 (sehr breit; $\mathrm{OH}$ ), 7.2-7.8 (m; $\mathrm{C}_{6} \mathrm{H}_{5}$ ).

$\mathrm{C}_{15} \mathrm{H}_{13} \mathrm{NO}_{5}(287.3)$ Ber. C 62.71 H 4.56 N 4.88 Gef. C 62.99 H 4.99 N 5.08

Dicarbonsäure-dimethylester 10a

a) Aus 9a: Man kochte $70 \mathrm{mg}(0.26 \mathrm{mmol}) 9 \mathrm{a}$ in $3 \mathrm{ml}$ absol. Methanol und 2 Tropfen konz. Schwefelsäure $6 \mathrm{~h}$ unter Rückfluß und goß dann auf $5 \mathrm{~g}$ Eis, wobei sich $30 \mathrm{mg}(37 \%)$ 10a als farblose Kristalle mit Schmp. $176-177^{\circ} \mathrm{C}$ abschieden.

b) Aus 8a: Man bereitete aus $1.00 \mathrm{~g}(9.70 \mathrm{mmol})$ Nitrosomethylharnstoff mit Kalilauge eine Lösung von Diazomethan in wasserhaltigem Ether, gab bei $0^{\circ} \mathrm{C} 150 \mathrm{mg}(0.56 \mathrm{mmol})$ $8 \mathrm{a}$ zu, rührte 24 h bei $20^{\circ} \mathrm{C}$ unter LichtausschluB und engte dann zum Teil ein. Dabei fielen $84 \mathrm{mg}(48 \%) 10$ a mit Schmp. $173-175^{\circ} \mathrm{C}$ aus; Umlösen aus Methanol ergab $60 \mathrm{mg}$ (34\%) mit Schmp. $177-178^{\circ} \mathrm{C}$. - IR (KBr): $1735,1720 \mathrm{~cm}^{-1}(\mathrm{C}=\mathrm{O}) .-\mathrm{MS}(70 \mathrm{eV}): m / e=315$ $\left(3 \%, \mathrm{M}^{+}\right), 145(100) .-{ }^{1} \mathrm{H}-\mathrm{NMR}\left(\mathrm{CDCl}_{3}\right): \delta=3.36$ (br. s; 2-, 5-H), 3.5-3.8 (m; 3-, 4-H), 3.72 (s; $2 \mathrm{CH}_{3}$ ), 4.40 (br. d, $J_{1,6}=6.0 \mathrm{~Hz} ; 1-\mathrm{H}$ ), 5.28 (br. d; 6-H), $7.3-7.8$ (m; $\mathrm{C}_{6} \mathrm{H}_{3}$ ).

$\mathrm{C}_{17} \mathrm{H}_{17} \mathrm{NO}_{5}$ (315.3) Ber. C 64.76 H 5.43 N 4.44 Gef. C 63.81 H 5.50 N 4.49

9-(2,4,6-Trimethylphenyl)-7-oxa-8-aza-exo-tricyclo[4.3.0.0.5 ]non-8-en-endo-3,endo-4-dicarbonsäureanhydrid (8b): Man rührte $290 \mathrm{mg}$ (1.93 mmol) 7 und $311 \mathrm{mg}$ (1.93 mmol) 2,4,6Trimethylbenzonitriloxid" $12 \mathrm{~h}$ bei $20^{\circ} \mathrm{C}$ in $4 \mathrm{ml}$ Dichlormethan, filtrierte die entstandenen farblosen Kristalle von $\mathbf{8 b}$ ab und wusch sie mit eiskaltem Dichlormethan. Nach dem Trocknen wogen sie $410 \mathrm{mg}(68 \%)$ und schmolzen bei $278-280^{\circ} \mathrm{C}$. - IR (KBr): 1855,1797 , $1777 \mathrm{~cm}^{-1}(\mathrm{C}=\mathrm{O}) .-\mathrm{MS}(70 \mathrm{eV}): \mathrm{m} / \mathrm{e}=311\left(19 \%, \mathrm{M}^{+}\right), 158(100)$.

$\mathrm{C}_{18} \mathrm{H}_{17} \mathrm{NO}_{4}$ (311.3) Ber. C 69.44 H 5.50 N 4.50 Gef. C 68.97 H 5.51 N 4.29

Dicarbonsäure $9 \mathrm{~b}$ : Aus $8 \mathrm{~b}$ entsprechend der Vorschrift bei 9a, Ausb. 71\%. Nach Umlösen aus Aceton/Wasser (1:2) lagen farblose Würfel vor, aus denen bei $220^{\circ} \mathrm{C}$ lange dünne Nadeln mit Schmp. $268-270^{\circ} \mathrm{C}$ wuchsen. Vermutlich deutet die Kristallumwandlung die Rückbildung des Anhydrids 8b an. - IR (KBr): 3700-2300 (breit, $\mathrm{O}-\mathrm{H}), 1720,1692 \mathrm{~cm}^{-1}(\mathrm{C}=\mathrm{O})$. - MS (70 eV): Übereinstimmend mit MS von 8b. - ${ }^{1} \mathrm{H}-\mathrm{NMR}\left[\left(\mathrm{CD}_{3}\right)_{2} \mathrm{CO}\right]: \delta=2.25$ (s; $3 \mathrm{CH}_{3}$ ), 3.25 (br. s; 2-, 5-H), 3.67 (m; 3-, 4-H), 4.45 (br. d, $J_{1,6}=6.0 \mathrm{~Hz} ; 1-\mathrm{H}$ ), 5.35 (br. d; 6-H), 6.92 (br. s; 2 aromat. $\mathrm{H}$ ).

Dicarbonsäure-dimethylester $10 \mathrm{~b}$ : Aus $8 \mathrm{~b}$ entsprechend Vorschrift a) bei 10a; jedoch kristallisierte 10b nach Mischung der Reaktionslösung mit Eis nicht, so daß man mit Dichlormethan extrahierte, die organische Phase mit $\mathrm{NaHCO}_{3}$-Lösung entsäuerte und mit $\mathrm{Na}_{2} \mathrm{SO}_{4}$ trocknete. Die nach dem Einengen verbliebenen farblosen Kristalle wurden zweimal aus Methanol umgelöst, wobei $50 \% 10 \mathrm{~b}$ mit Schmp. $146^{\circ} \mathrm{C}$ anfielen. - IR (KBr): 1745 (sh), $1734,1724(\mathrm{sh}) \mathrm{cm}^{-1}(\mathrm{C}=\mathrm{O})$. - MS (70 eV): $m / e=357\left(9 \%, \mathrm{M}^{+}\right), 158(100)$. - ${ }^{1} \mathrm{H}-\mathrm{NMR}$

Chem. Ber. 118 (1985) 
$\left(\mathrm{CDCl}_{3}\right): \delta=2.27\left(\mathrm{~s} ; 3 \mathrm{CCH}_{3}\right), 3.1-3.7(\mathrm{~m} ; 2-, 3-, 4,5-\mathrm{H}), 3.63$ (s) und 3.68 (s) $\left(2 \mathrm{OCH}_{3}\right)$, 4.18 (br. d, $J_{1,6}=6.0 \mathrm{~Hz} ; 1-\mathrm{H}$ ), 5.22 (br. d; 6-H), 6.88 (br. s; 2 aromat. $\mathrm{H}$ ).

7,9-Diphenyl-7,8-diaza-exo-tricyclo[4.3.0.02.5]non-8-en-endo-3,endo-4-dicarbonsäureanhydrid (8c): $\mathrm{Zu} 1.51 \mathrm{~g}$ (10.0 mmol) 7 und $2.31 \mathrm{~g}$ (10.0 mmol) ( $\alpha$-Chlorbenzyliden)phenylhydrazin $^{11)}$, die bei $40-45^{\circ} \mathrm{C}$ in $30 \mathrm{ml}$ Benzol gelöst worden waren, tropfte man unter Rühren und Kühlung mit Eis innerhalb von $90 \mathrm{~min} 5.06 \mathrm{~g}(50.0 \mathrm{mmol})$ Triethylamin in $50 \mathrm{ml} \mathrm{Benzol}$. Danach wurde $1 \mathrm{~h}$ bei $20^{\circ} \mathrm{C}$ gerührt, der Niederschlag abfiltriert, mit Benzol gewaschen, mit Wasser digeriert, erneut abfiltriert, mit eiskaltem Ether gewaschen und getrocknet: $1.54 \mathrm{~g}$ $(45 \%) 8 \mathrm{c}$ als gelbe Kristalle mit Schmp. $269-270^{\circ} \mathrm{C}$, deren Lösung stark fluoresziert; Schmp. nach Umlösen aus Aceton/Petrolether $\left(30-50^{\circ} \mathrm{C}\right) 270-272^{\circ} \mathrm{C}$. - IR (KBr): 1860,1790 $(\mathrm{C}=\mathrm{O}), 1600 \mathrm{~cm}^{-1}(\mathrm{C}=\mathrm{N}) .-\mathrm{UV}\left(\mathrm{CH}_{3} \mathrm{CN}\right): \lambda_{\max }(\log \varepsilon)=368$ (4.20), 309 (3.68), 254 (4.06), 243 (sh) nm (4.05). - MS (70 eV): $m / e=344\left(13 \%, \mathrm{M}^{+}\right), 220(100) .-{ }^{1} \mathrm{H}-\mathrm{NMR}\left[\left(\mathrm{CD}_{3}\right)_{2} \mathrm{CO}\right]$ : $\delta=3.28$ (br. s; 2-, 5-H), 4.17 (br. s; 3-, 4-H), 4.85 (br. d, $J_{1,6}=7.5 \mathrm{~Hz} ; 1-\mathrm{H}$ ), 5.28 (br. d; 6-H), $6.7-8.0\left(\mathrm{~m} ; 2 \mathrm{C}_{6} \mathrm{H}_{5}\right)$.

Dicarbonsäure 9c: Aus 8c entsprechend der Vorschrift bei 9a, Ausb. 98\%. Nach Umlösen aus Aceton/Wasser (1:2) lag der Schmp. der gelben Kristalle bei $262-263^{\circ} \mathrm{C}$; die Lösung zeigte starke Fluoreszenz. - IR (KBr): $3700-2300$ (breit, O-H), 1715 (breit, C=O), 1600 $\mathrm{cm}^{-1}(\mathrm{C}=\mathrm{N})$. - MS (70 eV): Übereinstimmend mit MS von 8c. - ${ }^{1} \mathrm{H}-\mathrm{NMR}$ [(C.D $\left.)_{2} \mathrm{SO}\right]:$ $\delta=3.02$ (br. s; 2-, 5-H), 3.90 (br. s; 3-, 4-H), 4.55 (br. d, $J_{1.6}=7.5 \mathrm{~Hz} ; 1-\mathrm{H}$ ), 5.05 (br. d; 6-H), 6.7-7.8 (m;2 $\left.\mathrm{C}_{6} \mathrm{H}_{5}\right)$.

$$
\mathrm{C}_{21} \mathrm{H}_{18} \mathrm{~N}_{2} \mathrm{O}_{4}(362.4) \text { Ber. C } 69.60 \text { H } 5.01 \text { N } 7.73 \text { Gef. C } 70.65 \text { H } 5.16 \text { N } 7.59
$$

Dicarbonsäure-dimethylester 10c: Aus 8c entsprechend der Vorschrift a) bei 10a; nach Umlösen aus Methanol erhielt man $62 \%$ hellgelbes $10 \mathrm{c}$ mit Schmp. $178-179^{\circ} \mathrm{C}$. In Chloroform veränderte sich die Farbe sehr rasch nach Dunkelgrün, in anderen Lösungsmitteln dauerte die Farbänderung einige Tage. - IR $(\mathrm{KBr}): 1743,1724(\mathrm{C}=\mathrm{O}), 1597 \mathrm{~cm}^{-1}(\mathrm{C}=\mathrm{N})$. - MS (70 eV): $m / e=390\left(6 \%, \mathrm{M}^{+}\right), 220(100) .-{ }^{1} \mathrm{H}-\mathrm{NMR}\left[\left(\mathrm{CD}_{3}\right)_{2} \mathrm{CO}\right]: \delta=3.23$ (br. $\mathrm{s}$; 2-, 5-H), 3.64 (s; $\left.2 \mathrm{CH}_{3}\right), 4.04$ (m; 3-, 4-H), 4.66 (br. d, $\left.J_{1,6}=7.5 \mathrm{~Hz} ; 1-\mathrm{H}\right), 5.13$ (br. d; 6-H), $6.7-7.9\left(\mathrm{~m} ; 2 \mathrm{C}_{6} \mathrm{H}_{5}\right)$.

$\mathrm{C}_{23} \mathrm{H}_{22} \mathrm{~N}_{2} \mathrm{O}_{4}(390.4)$ Ber. C 70.76 H 5.68 N 7.18 Gef. C 69.92 H 6.04 N 6.95

7,8-Diaza-exo-tricyclo[4.3.0.0.5.5 /non-7-en-endo-3,endo-4-dicarbonsäure-dimethylester (10d): Man bereitete aus $5.00 \mathrm{~g}$ (48.5 mmol) Nitrosomethylharnstoff mit Kalilauge eine Lösung von Diazomethan in wasserhaltigem Ether, gab unter Kühlung mit Eis portionsweise $600 \mathrm{mg}$ ( $4.80 \mathrm{mmol}) 7 \mathrm{zu}$ und bewahrte die entstandene Lösung $1 \mathrm{~d}$ bei $20^{\circ} \mathrm{C} \mathrm{im}$ Dunkeln auf. Durch einige Tropfen Essigsäure vernichtete man das noch verbliebene Diazomethan, filtrierte einen Niederschlag ab und engte i.Vak. ein. Aus dem gelben, festen Rückstand gewann man durch Umlösen aus Essigester $575 \mathrm{mg}(60 \%) 10 d$ in farblosen Kristallen mit Schmp. $106-107^{\circ} \mathrm{C}$ - I IR (KBr): 1726, 1718 (sh) $\mathrm{cm}^{-1}(\mathrm{C}=0)$. - UV $\left(\mathrm{CH}_{3} \mathrm{CN}\right): \lambda_{\max }(\log \varepsilon)=322 \mathrm{~nm}(2.57) .-\mathrm{MS}(70 \mathrm{eV}): m / e=238\left(2 \%, \mathrm{M}^{+}\right), 91$ (100). - ' $\mathrm{H}-\mathrm{NMR}$ : Tab. 1. - ${ }^{13} \mathrm{C}-\mathrm{NMR}\left(\mathrm{CDCl}_{3}\right): \delta=35.58$ (d, $145.3 \mathrm{~Hz}$; C-1), 42.22 (d, $154.0 \mathrm{~Hz}$ ) und $42.69(\mathrm{~d}, 152.6 \mathrm{~Hz})(\mathrm{C}-2,-5), 44.37(\mathrm{~d}, 141.0 \mathrm{~Hz})$ und $46.12(\mathrm{~d}, 139.5 \mathrm{~Hz})$ (C-3, -4), 52.01 und 52.06 (jeweils q, $146.8 \mathrm{~Hz} ; 2 \mathrm{CH}_{3}$ ), 83.77 (t, $142.4 \mathrm{~Hz}$; C-9), 92.67 (d, $155.5 \mathrm{~Hz}$; C-6), 171.99 und 172.19 (jeweils s; $2 \mathrm{C}=\mathrm{O}$ ).

$\mathrm{C}_{11} \mathrm{H}_{14} \mathrm{~N}_{2} \mathrm{O}_{4}$ (238.2) Ber. C 55.46 H $5.92 \mathrm{~N} 11.76$ Gef. C 55.19 H 5.96 N 11.80

1,8,11,12-Tetrachlor-9,10-dioxotetracyclo[6.2.2.0.7.7.03.6 ]dodec-11-en-4,5-dicarbonsäureanhydrid (11): $2.00 \mathrm{~g}$ (13.3 mmol) 7 und $3.30 \mathrm{~g}$ (13.3 mmol) o-Chloranil wurden in $50 \mathrm{ml}$ absol. Dichlormethan $5 \mathrm{~d}$ unter RückfluB gekocht. Es bildeten sich $1.60 \mathrm{~g} \mathrm{(30 \% )} 11$ als gelber Niederschlag, der abfiltriert und mit Aceton und Ether gewaschen wurde. Das 
Pulver wandelte sich bei $264-265^{\circ} \mathrm{C}$ in dünne Stäbchen um, die bei $327-329^{\circ} \mathrm{C}$ (Zers.) schmolzen. - IR (KBr): 1855, 1780 (breit) $\mathrm{cm}^{-1}(\mathrm{C}=\mathrm{O})$. - UV $\left(\mathrm{CH}_{2} \mathrm{Cl}_{2}\right): \lambda_{\max }(\log \varepsilon)=$ 436 (2.57), 286 (3.23), $240 \mathrm{~nm}(3.46) .-\mathrm{MS}(70 \mathrm{eV}): m / e=344,342,340,338(1 \%, 4 \%$, $\left.7 \%, 6 \%, \mathrm{M}^{+}-2 \mathrm{CO}\right), 220,218,216,214\left(11,48,100,79, \mathrm{C}_{6} \mathrm{H}_{2} \mathrm{Cl}_{4}\right) .-{ }^{1} \mathrm{H}-\mathrm{NMR}$ [(CD $\left.)_{2} \mathrm{CO}\right]: \delta=2.75$ (br. s; 3-H), 3.87 und 3.95 (jeweils br. s; 2- und 4-H).

$$
\mathrm{C}_{14} \mathrm{H}_{6} \mathrm{Cl}_{4} \mathrm{O}_{5} \text { (396.0) Ber. C } 42.46 \mathrm{H} 1.53 \text { Gef. C } 42.43 \text { H } 1.72
$$

7,8,9,10-Tetrachlortricyclo[4.4.0.0 2.5] deca-7,9-dien-3,4-dicarbonsäureanhydrid (12): $135 \mathrm{mg}$ $(0.90 \mathrm{mmol}) 7$ und $230 \mathrm{mg}(0.90 \mathrm{mmol})$ Tetrachlorthiophen-1,1-dioxid $\left.{ }^{13}\right)$ wurden in $0.5 \mathrm{ml} \mathrm{CDCl} 24 \mathrm{~h}$ auf $40-50^{\circ} \mathrm{C}$ erwärmt. Es fielen $210 \mathrm{mg}(69 \%) 12$ als bräunliche Kristalle mit Schmp. $224-226^{\circ} \mathrm{C}$ aus. - IR (KBr): $1860,1782(\mathrm{C}=\mathrm{O}), 1615 \mathrm{~cm}^{-1}(\mathrm{C}=\mathrm{C})$. - MS $(70 \mathrm{eV}): m / e=344,342,340,338\left(1 \%, 4 \%, 8 \%, 6 \%, \mathrm{M}^{+}\right), 220,218,216,214(10$, 48, 100, 78, $\left.\mathrm{C}_{6} \mathrm{H}_{2} \mathrm{Cl}_{4}\right) .-{ }^{1} \mathrm{H}-\mathrm{NMR}\left[\left(\mathrm{CD}_{3}\right)_{2} \mathrm{CO}\right]: \delta=3.51$ (br. s; $\left.2-\mathrm{H}\right), 4.05$ und 4.22 (jeweils br. s; 1- und 3-H).

$$
\mathrm{C}_{12} \mathrm{H}_{6} \mathrm{Cl}_{4} \mathrm{O}_{3}(340.0) \quad \text { Ber. } \mathrm{Cl} 41.71 \text { Gef. } \mathrm{Cl} 41.33
$$

\section{B. Synthese von und 1,3-dipolare Cycloadditionen an Dewarbenzol (16)}

$N$-Methyl-7,8-diazatetracyclo[3.3.0.0.0.4. $0^{3.6}$ Joctan-7,8-dicarboximid (13): $250 \mathrm{ml}$ einer Lösung von Benzvalen ${ }^{26,27}(8.59 \mathrm{~g}, 110 \mathrm{mmol})$ in Ether wurden mit $200 \mathrm{ml} \mathrm{absol}$. Dichlormethan verdünnt, auf $0^{\circ} \mathrm{C}$ gekühlt und unter Stickstoff portionsweise mit $12.5 \mathrm{~g} \mathrm{(110}$ mmol) 4-Methyl-4H-1,2,4-triazol-3,5-dion versetzt. Unter Rühren wartete man jeweils ab, bis die Rotfärbung ausgebleicht war, und gab dann erst die nächste Portion zu (Gesamtdauer $60 \mathrm{~min}$ ). Am Ende rührte man noch $30 \mathrm{~min}$ bei $22^{\circ} \mathrm{C}$, entfernte die Lösungsmittel i. Vak. und ermittelte im Rückstand, einem gelbbraunen $\mathrm{Ol}$, durch ein ${ }^{1} \mathrm{H}-\mathrm{NMR}$-Spektrum mit Mesitylen als internem Standard die Ausbeute an $13 \mathrm{zu} 99 \%$. Chromatographie an Kieselgel (Säule $30 \times 2.5 \mathrm{~cm}$ ) mit Chloroform lieferte $11.6 \mathrm{~g}(55 \%) 13$ als gelbliche Kristalle mit Schmp. $113^{\circ} \mathrm{C}$. - IR (KBr): $1780,1700 \mathrm{~cm}^{-1}(\mathrm{C}=\mathrm{O}) . \quad{ }^{1} \mathrm{H}-\mathrm{NMR}\left(\mathrm{CDCl}_{3}\right): \delta=2.35$ $(\mathrm{m} ; 2-, 3-\mathrm{H}), 2.67\left(\mathrm{td}, J_{2,4}=3.3, J_{4,5}=1.1 \mathrm{~Hz} ; 4-\mathrm{H}\right), 3.01\left(\mathrm{~s} ; \mathrm{CH}_{3}\right.$ ), 3.15 (quint d, $J_{1,5}=J_{2,5}=$ $4.5 \mathrm{~Hz} ; 5-\mathrm{H}$ ), 5.00 (d des $\mathrm{AA}^{\prime}$-Teils eines $\mathrm{AA}^{\prime} \mathrm{XX}^{\prime}$-Spektrums, $J_{1,2}=2.7 \mathrm{~Hz} ; 1-, 6-\mathrm{H}$ ). ${ }^{13}$ C-NMR: Lit. ${ }^{28)}$

N-Methyl-7,8-diazatetracyclo/3.3.0.0.0.4.0.0 Joctan-7-carboxamid (14): Unter Stickstoff kochte man $1.27 \mathrm{~g}(6.64 \mathrm{mmol}) 13$ und $1.75 \mathrm{~g}$ (31.3 mmol) KOH in $25 \mathrm{ml}$ absol. Methanol $6 \mathrm{~h}$ unter Rückfluß. Das erkaltete Gemisch wurde filtriert und i. Vak. vom Solvens befreit. Man nahm den Rückstand in $20 \mathrm{ml}$ Wasser auf, neutralisierte bei $0^{\circ} \mathrm{C}$ mit konz. Salzsäure, filtrierte, extrahierte das Filtrat fünfmal mit je $30 \mathrm{ml}$ Dichlormethan, trocknete die organische Phase über $\mathrm{MgSO}_{4}$ und engte i.Vak. ein, wobei $693 \mathrm{mg}(63 \%) 14$ als ockerfarbene Kristalle mit Schmp. $94-95^{\circ} \mathrm{C}$ zurückblieben. Zweimaliges Lösen in warmem Dichlormethan und Ausfällen mit Hexan lieferte $575 \mathrm{mg}(52 \%)$ gelbliche Kristalle mit Schmp. $97-98^{\circ} \mathrm{C}$. - IR (KBr): 3373, 3200 (N-H), 1641, $1545 \mathrm{~cm}^{-1}$ (Amid). - MS (70 eV): $m / e=165$ (2\%, $\mathrm{M}^{+}$), 80 (100). - ' $\mathrm{H}-\mathrm{NMR}\left(\mathrm{CDCl}_{3}\right): \delta=1.94\left(\mathrm{tdd}, J_{1,2}=2.5, J_{2,3}=J_{2,5}=4.5, J_{24}=\right.$ $3.4 \mathrm{~Hz} ; 2-\mathrm{H}$ ), 2.21 (tdd, $J_{3.4}=3.4, J_{3,5}=4.5, J_{3,6}=2.2 \mathrm{~Hz} ; 3-\mathrm{H}$ ), 2.44 (td, $J_{4.5}=1.0 \mathrm{~Hz}$; 4-H), $2.81\left(\mathrm{~d}, J_{\mathrm{NH}_{1} \mathrm{CH}_{3}}=5.0 \mathrm{~Hz} ; \mathrm{CH}_{3}\right), 2.96$ (br. quint; 5-H), 4.16 (breit; 8-H), 4.24 (dd, $J_{1,5}=$ $4.5 \mathrm{~Hz} ; 1-\mathrm{H}$ ), 4.89 (dd, $\left.J_{5.6}=4.5 \mathrm{~Hz} ; 6-\mathrm{H}\right), 6.23$ (breit, $\mathrm{NHCH}_{3}$ ). $-{ }^{13} \mathrm{C}-\mathrm{NMR}: \mathrm{Lit}^{28)}$.

\section{$\mathrm{C}_{8} \mathrm{H}_{11} \mathrm{~N}_{3} \mathrm{O}(165.2) \quad$ Ber. C 58.16 H 6.71 N 25.44 Gef. C 58.30 H 6.97 N 25.18}

7,8-Diazatetracyclo[3.3.0.0 $0^{2.4} .0^{3,6}$ ]oct-7-en (17): Unter Stickstoff kochte man $11.5 \mathrm{~g}(60.2$ $\mathrm{mmol}) 13$ und $16.0 \mathrm{~g}$ (285 mmol) $\mathrm{KOH}$ in $150 \mathrm{ml}$ absol. Methanol $24 \mathrm{~h}$ unter Rückfluß. Das erkaltete Gemisch wurde filtriert, das Filtrat mit konz. Salzsäure auf pH 2 gebracht, erneut filtriert und mit konz. Ammoniak auf pH 5-6 eingestellt. Nun gab man $90 \mathrm{ml} 3 \mathrm{~N}$ 
$\mathrm{CuCl}_{2}$ in Wasser $\mathrm{zu}$, bewahrte 2-3 d im Kühlschrank auf, filtrierte den schwarzen Niederschlag ab und wusch ihn nacheinander mit gesättigter NaCl-Lösung, Methanol und Wasser. In trockenem Zustand waren die Kristalle explosiv, weshalb sie feucht gehalten wurden. Man löste sie in konz. Ammoniak, extrahierte mehrmals mit Dichlormethan, trocknete die organische Phase mit $\mathrm{Na}_{2} \mathrm{SO}_{4}$ und ermittelte durch ein ${ }^{1} \mathrm{H}-\mathrm{NMR}$-Spektrum mit Mesitylen als internem Standard die Ausbeute zu $4.09 \mathrm{~g}(64 \%) 17$, gelōst in $250 \mathrm{ml}$ Dichlormethan. ${ }^{1} \mathrm{H}-\mathrm{NMR}$ : Stimmt mit Literaturangaben ${ }^{17}$ überein. $-{ }^{13} \mathrm{C}-\mathrm{NMR}$ : Lit. ${ }^{28)}$.

Dewarbenzol (16): Man gab $80 \mathrm{ml}$ absol. Tetrachlorethen zu $3.90 \mathrm{~g}(36.8 \mathrm{mmol}) 17$ in $120 \mathrm{ml}$ Dichlormethan, destillierte i.Vak. das Dichlormethan ab, sättigte die zurückbleibende 17-Lösung mit Stickstoff und belichtete bei $20^{\circ} \mathrm{C} 10 \mathrm{~h}$ mit einer $\mathrm{Hg}$-Mitteldrucklampe (Hanovia 450 Watt) durch Pyrex. Im Abstand von jeweils $1 \mathrm{~h}$ unterbrach man, um vom Tauchschacht einen Belag abzuschaben. Das Fortschreiten der Reaktion wurde anhand von NMR-Spektren überwacht. Nach vollständiger Umsetzung kondensierte man die flüchtigen Anteile zusammen mit einem Teil des Tetrachlorethens bei 16 Torr unter leichtem Erwärmen in eine auf $-78^{\circ} \mathrm{C}$ gekühlte Vorlage. Das ${ }^{1} \mathrm{H}-\mathrm{NMR}$-Spektrum des Kondensats glich, von fehlenden 1,8-Diazacyclooctatetraen-Signalen abgesehen, einem in Lit. ${ }^{16 \mathrm{e})}$ abgebildeten. Mit Mesitylen als internem Standard wurde die Ausbeute ermittelt. In typischen Fällen ergaben sich $314 \mathrm{mg}(11 \%)$ und $258 \mathrm{mg}(9 \%) 16$ in wechselnden Mengen (11-48 ml) Tetrachlorethen.

9-Phenyl-7-oxa-8-aza-exo-tricyclo[4.3.0.0.0.5 nona-3,8-dien (6a) und 5,11-Diphenyl-3,9dioxa-4,10-diaza-exo,exo-tetracyclo[5.5.0.0 $2.6 .0^{8,12}$ ]dodeca-4,10-dien (18a): $\mathrm{Zu} 260 \mathrm{mg}$ (3.33 mmol) Dewarbenzol (16) und $512 \mathrm{mg}(3.30 \mathrm{mmol})$ Benzhydroximoylchlorid ${ }^{25}$ in $11 \mathrm{ml}$ Tetrachlorethen unter Stickstoff tropfte man bei $0^{\circ} \mathrm{C}$ unter Rühren innerhalb von $4 \mathrm{~h} 1.5 \mathrm{ml}(10.9$ mmol) Triethylamin in $8.5 \mathrm{ml}$ Tetrachlorethen. Der Niederschlag wurde abfiltriert, das Filtrat i.Vak. eingeengt und der Rückstand über Kieselgel (Säule $20 \times 2.5 \mathrm{~cm}$ ) mit Toluol chromatographiert. Dabei fielen $312 \mathrm{mg}$ rohes 6a (kürzere Retentionszeit) als gelbes Öl und $170 \mathrm{mg}$ rohes 18a (längere Retentionszeit) als farblose Kristalle an. Das 1:1-Addukt 6a wurde bei $60-80^{\circ} \mathrm{C}(\mathrm{Bad}) / 0.001$ Torr destilliert und ergab auf Zusatz von Pentan $250 \mathrm{mg}$ (38\%) farblose Kristalle mit Schmp. $44-45^{\circ} \mathrm{C}$. MS (70 eV): $m / e=197\left(4 \%, \mathrm{M}^{+}\right), 78$ (100). $-{ }^{1} \mathrm{H}-\mathrm{NMR}\left(\mathrm{CDCl}_{3}\right): \delta=3.58(\mathrm{~m} ; 2-\mathrm{H}), 3.72$ (br. t, $\left.J_{2,5}=J_{4,5}=2.3 \mathrm{~Hz} ; 5-\mathrm{H}\right), 4.23$ (dd, $\left.J_{1,2}=1.1, J_{1,6}=6.0 \mathrm{~Hz} ; 1-\mathrm{H}\right), 5.15$ (br. d; 6-H), 6.35 (br. t, $J_{2,3}=J_{3,4}=2.3 \mathrm{~Hz} ; 3-\mathrm{H}$ ), 6.47 (br. t; 4-H), $\mathrm{C}_{6} \mathrm{H}_{5}: 7.35-7.60(\mathrm{~m} ; 3 \mathrm{H}), 7.60-7.85(\mathrm{~m} ; 2 \mathrm{H}) .-{ }^{13} \mathrm{C}-\mathrm{NMR}\left(\mathrm{CDCl}_{3}\right): \delta=$ 49.74 (d, $155.5 \mathrm{~Hz}$ ) und 53.77 (d, $159.0 \mathrm{~Hz}$ (C-2 und C-5), 53.28 (d, $147.8 \mathrm{~Hz}$, C-1), 84.95 (d, 167.7 Hz; C-6), 140.11 (d, $172.1 \mathrm{~Hz})$ und 142.68 (d, $173.0 \mathrm{~Hz}$ ) (C-3 und C-4), 156.23 (s; C-9), $\mathrm{C}_{6} \mathrm{H}_{5}: 126.76$ und 128.74 (jeweils d; $\left.o-, m-C\right), 128.65$ (s; ipso-C), 129.91 (d; $p-C$ ).

\section{$\mathrm{C}_{13} \mathrm{H}_{11} \mathrm{NO}$ (197.2) Ber. C 79.16 H 5.62 N 7.10 Gef. C 79.08 H 5.62 N 6.84}

Das 1:2-Addukt 18a wurde in Chloroform gelöst und mit Pentan wieder ausgefältt, woraus $110 \mathrm{mg}(21 \%) \mathrm{mit}$ Schmp. $240-243^{\circ} \mathrm{C}$ (Zers.) hervorgingen. 18a konnte bei $180-190^{\circ} \mathrm{C} / 0.2$ Torr sublimiert werden. $-\mathrm{MS}(70 \mathrm{eV}): \mathrm{m} / \mathrm{e}=316\left(12 \%, \mathrm{M}^{+}\right), 145$ (100). - 'H-NMR $\left(\mathrm{CDCl}_{3}\right): \delta=3.37(\mathrm{~s} ; 1-\mathrm{H}), 4.42\left(\mathrm{~d}, J_{2,6}=6.0 \mathrm{~Hz} ; 6-\mathrm{H}\right), 5.43(\mathrm{~d} ; 2-\mathrm{H})$, $\mathrm{C}_{6} \mathrm{H}_{5}: 7.35-7.52(\mathrm{~m} ; m-, p-\mathrm{H}), 7.64(\mathrm{~m} ; o-\mathrm{H}),-{ }^{13} \mathrm{C}-\mathrm{NMR}\left(\mathrm{CDCl}_{3}\right): \delta=49.57$ und 53.25 (C-1 und C-6), 84.14 (C-2), 157.70 (C-5), $\mathrm{C}_{6} \mathrm{H}_{5}: 126.96$ und 128.97 (o-, m-C), 127.29 (ipso-C), $130.50(p-\mathrm{C})$.

9-(2,4,6-Trimethylphenyl)-7-oxa-8-aza-exo-tricyclo[4.3.0.0.0.5 Jnona-3,8-dien (6b), 5,11-Bis(2,4,6-trimethylphenyl)-3,9-dioxa-4,10-diaza-exo,exo-tetracyclo[5.5.0.0.0.6. $.0^{8.12}$ ] dodeca-4,10dien (18b) und 5,9-Bis(2,4,6-trimethylphenyl)-3,11-dioxa-4,10-diaza-exo,exo-tetracyclo[5.5.0.0 $0^{2,6} \cdot 0^{8,12}$ ]dodeca-4,9-dien (19b): Man rührte $280 \mathrm{mg}$ (3.59 mmol) 16 in $15 \mathrm{ml}$ Tetra- 
chlorethen und $573 \mathrm{mg}$ ( $3.56 \mathrm{mmol})$ 2,4,6-Trimethylbenzonitriloxid $\left.{ }^{9}\right) 3 \mathrm{~d}$ unter Stickstoff bei $22^{\circ} \mathrm{C}$. Man filtrierte den Niederschlag, der das $1: 2$-Addukt $18 \mathrm{~b}$ enthielt, ab und engte das Filtrat i. Vak. ein. Aus dem Rückstand destillierten bei $70-105^{\circ} \mathrm{C}(\mathrm{Bad}) / 0.001$ Torr $280 \mathrm{mg}$ eines gelben Ols, das mit Pentan zur Kristallisation gebracht wurde. Umlösen aus Hexan lieferte $190 \mathrm{mg}(22 \%) 6 \mathrm{~b}$ als farblose Kristalle mit Schmp. $93-94^{\circ} \mathrm{C}$. - MS (70 eV): $m / e=239\left(17 \%, \mathrm{M}^{+}\right), 210(99), 196(92), 91$ (97), $52(100) .-{ }^{1} \mathrm{H}-\mathrm{NMR}$ : Tab. 2 - ${ }^{13} \mathrm{C}-\mathrm{NMR}$ $\left(\mathrm{CDCl}_{3}\right): \delta=20.11\left(\mathrm{q}, 126.5 \mathrm{~Hz}, 0-\mathrm{CH}_{3}\right), 21.02\left(\mathrm{q}, 126.5 \mathrm{~Hz}_{;} \mathrm{p}-\mathrm{CH}_{3}\right), 48.70(\mathrm{~d}, 156 \mathrm{~Hz})$ und 54.41 (d, $159 \mathrm{~Hz}$ ) (C-2, -5), 57.44 (d, $149.3 \mathrm{~Hz} ; \mathrm{C}-1), 84.33$ (d, $165 \mathrm{~Hz}$; C-6), 125.40 (s; ipsoC), $128.74(\mathrm{~d}, 157 \mathrm{~Hz} ; m-\mathrm{C}), 136.80(\mathrm{~s} ; o-\mathrm{C}), 138.62(\mathrm{~s} ; p-\mathrm{C}), 140.08(\mathrm{~d}, 170 \mathrm{~Hz})$ und 142.62 $(\mathrm{d}, 175 \mathrm{~Hz})(\mathrm{C}-3,-4), 156.65$ (s; C-9).

$\mathrm{C}_{16} \mathrm{H}_{17} \mathrm{NO}(239.3)$ Ber. C 80.30 H 7.16 N 5.85 Gef. C 80.40 H 7.28 N 5.78

Der $18 \mathrm{~b}$ enthaltende Niederschlag wurde über Kieselgel (Säule $10 \times 1 \mathrm{~cm}$ ) mit Dichlormethan chromatographiert, wobei $220 \mathrm{mg}(31 \%) 18 \mathrm{~b}$ als farblose Kristalle mit Schmp. 272-274 ${ }^{\circ} \mathrm{C}$, nach Umlösen aus Benzol $275-277^{\circ} \mathrm{C}$, anfielen. $-\mathrm{MS}(70 \mathrm{eV}): \mathrm{m} / \mathrm{e}=400$ $\left(16 \%, \mathrm{M}^{\prime}\right), 158(100)$ - ' $\mathrm{H}-\mathrm{NMR}\left(\mathrm{CDCl}_{3}\right): \delta=2.27\left(\mathrm{~s} ; o-\mathrm{CH}_{3}\right), 2.30\left(\mathrm{~s} ; p-\mathrm{CH}_{3}\right), 3.32(\mathrm{~s}$; 1-H), $4.20\left(\mathrm{~d}, J_{2,6}=6.0 \mathrm{~Hz} ; 6-\mathrm{H}\right), 5.20(\mathrm{~d} ; 2-\mathrm{H}), 6.92(\mathrm{~s} ; m-\mathrm{H}) .-{ }^{13} \mathrm{C}-\mathrm{NMR}\left(\mathrm{CDCl}_{3}\right): \delta=19.93$ (q; o- $\mathrm{CH}_{3}$ ), 21.05 (q; p-CH $\mathrm{CH}_{3}, 49.34$ (d; C-1), 57.17 (d; C-6), 83.41 (d; C-2), 124.50 (s; ipso-C), 128.90 (d; $m-\mathrm{C}), 136.68$ (s; $o-\mathrm{C}), 139.12$ (s; $p-\mathrm{C}), 157.91$ (s; C-5).

$\mathrm{C}_{26} \mathrm{H}_{28} \mathrm{~N}_{2} \mathrm{O}_{2}(400.5)$ Ber. C 77.97 H 7.05 N 6.99 Gef. C 78.09 H 7.09 N 6.75

Das ${ }^{1} \mathrm{H}$-NMR-Spektrum des $18 \mathrm{~b}$ enthaltenden Niederschlags in $\mathrm{C}_{6} \mathrm{D}_{6}$ enthielt über die Signale von $18 \mathrm{~b}$ hinaus Absorptionen, die sehr gut auf 19b passen, Verhältnis 18b:19b = 70:30. 18 b: $\delta=2.17$ und/oder 2.22 (jeweils s; $\mathrm{CH}_{3}$ ), 3.37 (br.s; $1-\mathrm{H}$ ), 3.68 (br.d; 6-H), 4.69 (br.d; $2-\mathrm{H}$ ), 6.77 (br.s; $m$-H); 19 b: $\delta=1.99$ (s; $1 \mathrm{CH}_{3}$ ), 2.17 und/oder 2.22 (jeweils s; $2 \mathrm{CH}_{3}$ ), 3.72 (dd, $J_{2,6}=6.0, J_{6,7}=1.2 \mathrm{~Hz} ; 6-\mathrm{H}$ ), 4.75 (br.d; 2-H), 6.58 (br.m; $m$-H), die Signale von 1- und 7-H konnten nicht eindeutig lokalisiert werden.

7,8-Diaza-exo-tricyclo[4.3.0.0.0.5 Jnona-3,7-dien (20), 7,8-Diaza-endo-tricyclo[4.3.0.0.0.5]nona-3,7-dien (21), 3,4,9,10-Tetraaza-exo,exo-tetracyclo[5.5.0.0.0.6. $\left.0^{8,12}\right]$ dodeca-3,9-dien (22) und 3,4,10,11-Tetraaza-exo,exo-tetracyclo[5.5.0.0.6.6.0.12]dodeca-3,10-dien (23): $\mathrm{Zu} 360 \mathrm{mg}$ (4.61 mmol) 16 in $4 \mathrm{ml}$ Tetrachlorethen gab man unter Stickstoff bei -5 bis $-10^{\circ} \mathrm{C} 189 \mathrm{mg}$ $(4.50 \mathrm{mmol})$ Diazomethan in $3.6 \mathrm{ml}$ Ether, belie $6 \mathrm{~h}$ bei $0^{\circ} \mathrm{C}$, dann $3 \mathrm{~d}$ bei $22^{\circ} \mathrm{C}$ unter Lichtausschluß und stellte danach das Gemisch in eine Kühltruhe $\left(-25^{\circ} \mathrm{C}\right)$. Nach $30 \mathrm{~d}$ hatten sich $118 \mathrm{mg} \mathrm{(32 \% )} \mathrm{eines} \mathrm{farblosen} \mathrm{Niederschlags} \mathrm{mit} \mathrm{Schmp.} 75-76^{\circ} \mathrm{C}$ abgeschieden, der bei $80-90^{\circ} \mathrm{C}(\mathrm{Bad}) / 0.1$ Torr sublimiert werden konnte und der sich als 55:45-Gemisch aus den 1:2-Addukten 22 und 23 erwies. - IR $(\mathrm{KBr}): 1550 \mathrm{~cm}^{-1}(\mathrm{~s}, \mathrm{~N}=\mathrm{N}) .-\mathrm{MS}(70 \mathrm{eV})$ : $m / e=162\left(2 \%, \mathrm{M}^{+}\right), 91(100)$. $-{ }^{1} \mathrm{H}-\mathrm{NMR}\left(\mathrm{CDCl}_{3}\right): 22: \delta=2.39$ (br.s; 1-H), 2.88 (br.ddd, teilweise durch ein Signal von 23 überlagert, $J_{2,6}=5.2, J_{\text {sexo. } 6}=2.3 \mathrm{~Hz} ; 6-\mathrm{H}$ ), 4.60 (ddd, $\left.J_{2, \text { Sexo }}=3.2, J_{5,5}=18.5 \mathrm{~Hz} ; 5-\mathrm{H}_{\text {exo }}\right), 4.71$ (ddd, $J_{2, \text { Sendo }}=1.5, J_{\text {sendo }, 6}=8.8 \mathrm{~Hz} ; 5-\mathrm{H}_{\text {endo }}$ ), 5.35 $(\mathrm{m} ; 2-\mathrm{H}) ; 23: \delta=1.84$ (br.d, $\left.J_{1,7}=4.1 \mathrm{~Hz} ; 7-\mathrm{H}\right), 2.62$ (dddd, $J_{2,6}=5.2, J_{6 \text {, Sendo }}=9.0, J_{6 \text { sexo }}=$ $\left.2.5, J_{6,7}=1.5 \mathrm{~Hz} ; 6-\mathrm{H}\right), 2.89\left(\mathrm{dt}, J_{1,2}=1.3 \mathrm{~Hz} ; 1-\mathrm{H}\right), 4.50\left(\mathrm{ddd}, J_{2,5 \times x_{0}}=3.0, J_{5,5}=18.5 \mathrm{~Hz}\right.$; $5-\mathrm{H}_{\text {exo }}$ ), 4.70 (ddd, $J_{2 \text { Sendo }}=1.5 \mathrm{~Hz} ; 5-\mathrm{H}_{\text {endo }}$ ), $5.62(\mathrm{~m} ; 2-\mathrm{H}) .-{ }^{13} \mathrm{C}-\mathrm{NMR}\left(\mathrm{CDCl}_{3}\right): 22: \delta=$ 34.34 (d; C-6), 48.09 (d; C-1), 83.65 (t; C-5), 93.00 (d; C-2); $23: \delta=35.79$ (d; C-6), 47.07 (d; C-7), 49.16 (d; C-1), 83.89 (t; C-5), 91.69 (d; C-2).

$\mathrm{C}_{8} \mathrm{H}_{10} \mathrm{~N}_{4}(162.2)$ Ber. C 59.24 H 6.21 N 34.55 Gef. C 59.49 H 6.40 N 34.08

Die Mutterlauge obiger Kristalle wurde i. Vak. eingeengt, und aus dem Rückstand destillierten bei $40-50^{\circ} \mathrm{C}(\mathrm{Bad}) / 0.001$ Torr $199 \mathrm{mg}$ eines gelben $\mathrm{O}_{\mathrm{ls}}$, das sich an der Luft rasch über Weinrot nach Violett und dann innerhalb $1 \mathrm{~d}$ nach Schwarz verfärbte. Auch Lösungen in $\mathrm{CDCl}_{3}$ verfärbten sich sehr rasch, jedoch waren solche in $\mathrm{C}_{6} \mathrm{D}_{6}$ einigermaßen haltbar. Auf

Chem. Ber. 118 (1985) 
der Basis der ${ }^{1}$ H-NMR-Signale lag ein 79:8:7:6-Gemisch aus 20 und 21 (30\% Ausbeute) sowie 22 und 23 mit nur noch geringfügigen weiteren Verunreinigungen vor. - MS $(70 \mathrm{eV})$ : $m / e=120\left(3 \%, M^{+}\right), 91(100)$. $-{ }^{1} H-N M R\left(C_{6} D_{6}\right): 20: \delta=1.76$ (dddd, $J_{1,2}=1.3, J_{1,6}=$ $\left.5.0, J_{1,9 e n d o}=2.5, J_{1,9 e x o}=8.0 \mathrm{~Hz} ; 1-\mathrm{H}\right), 2.29$ (br.s; $\left.2-\mathrm{H}\right), 3.23$ (br.s; 5-H), 3.86 (ddd, $J_{6,9 e \times o}=$ $\left.1.6, J_{9,9}=18.0 \mathrm{~Hz} ; 9-\mathrm{H}_{\text {exo }}\right), 3.93\left(\approx \mathrm{dt}, J_{6,9 \text { endo }}=3.2 \mathrm{~Hz} ; 9-\mathrm{H}_{\text {endo }}\right), 4.96(\mathrm{~m} ; 6-\mathrm{H}), 5.92(\mathrm{td}$, $\left.J_{2,3}=J_{3,4}=2.3, J_{3,5}=0.6 \mathrm{~Hz} ; 3-\mathrm{H}\right), 5.96(\mathrm{t} ; 4-\mathrm{H}) .21: \mathrm{Tab} .3 .-{ }^{13} \mathrm{C}-\mathrm{NMR}\left(\mathrm{C}_{6} \mathrm{D}_{6}\right): 20: \delta=$ 34.43 (d; C-1), 50.61 und 50.96 (jeweils d; C-2, -5), 79.65 (t; C-9), 92.84 (d; C-6), 139.95 und 143.92 (jeweils d; C-3, -4).

\section{Die Thermolyse des Dewarbenzol-Addukts 6b}

a) Ohne Zusatz: Auf $50 \mathrm{mg}$ (0.209 mmol) 6b in einem dickwandigen NMR-Rohr kondensierte man i.Vak. $0.3 \mathrm{ml}$ trockenes $\mathrm{C}_{6} \mathrm{D}_{6}$, schmolz unter Vak. ab und erhitzte die Probe auf $120^{\circ} \mathrm{C}$. Mit Hilfe von ${ }^{1} \mathrm{H}-\mathrm{NMR}$-Spektren wurde der Verlauf überwacht und festgestellt, daß $6 \mathrm{~b}$ innerhalb von $8 \mathrm{~h}$ vollständig in Benzol sowie $18 \mathrm{~b}$ und $19 \mathrm{~b}$ im Verhältnis $2: 1$ überging.

b) In Gegenwart von Fumarsäure-dimethylester: Auf $44.4 \mathrm{mg}(0.185 \mathrm{mmol}) 6 \mathrm{~b}$ und $26.7 \mathrm{mg}$ ( $0.185 \mathrm{mmol})$ Fumarsäure-dimethylester in einem dickwandigen NMR-Rohr kondensierte man i. Vak. $0.3 \mathrm{ml}$ trockenes $\mathrm{C}_{6} \mathrm{D}_{6}$, schmolz unter Vak. ab und erhitzte die Probe auf $120^{\circ} \mathrm{C}$. Von Zeit zu Zeit aufgenommene 'H-NMR-Spektren zeigten ausschließlich die Bildung von Benzol und 24 an mit einer Halbwertszeit von ca. $3 \mathrm{~h}$.

3-(2,4,6-Trimethylphenyl)- $\Delta^{2}$-isoxazolin-4,5-trans-dicarbonsäure-dimethylester (24): $100 \mathrm{mg}$ (0.62 mmol) 2,4,6-Trimethylbenzonitriloxid und $70 \mathrm{mg}(0.49 \mathrm{mmol})$ Fumarsäure-dimethylester wurden in der gerade notwendigen Menge $\mathrm{CDCl}_{3}$ in einem NMR-Rohr gelöst und bei $20^{\circ} \mathrm{C}$ aufbewahrt. Von Zeit zu Zeit aufgenommene ${ }^{1} \mathrm{H}-\mathrm{NMR}-S p e k t r e n$ zeigten die Vollständigkeit der Umsetzung nach ca. $4 \mathrm{~h}$ an. Durch Einengen i. Vak. und Umlösen des festen Rückstands aus Methanol fielen ca. $100 \mathrm{mg}$ (67\%) farbloses 24 mit Schmp. $129-131^{\circ} \mathrm{C}$ an. - IR (KBr): $1763,1744 \mathrm{~cm}^{-1}(\mathrm{C}=\mathrm{O})$. - ${ }^{1} \mathrm{H}-\mathrm{NMR}\left(\mathrm{CDCl}_{3}\right): \delta=2.37\left(\mathrm{~s} ; o-\mathrm{CH}_{3}\right), 2.43$ (s; p- $\mathrm{CH}_{3}$ ), 3.71 und 3.94 (jeweils s; $\left.\mathrm{OCH}_{3}\right), 4.79\left(\mathrm{~d}, \mathrm{~J}_{4,5}=6.0 \mathrm{~Hz} ; 4 \mathrm{H}\right), 5.62(\mathrm{~d} ; 5-\mathrm{H}), 6.90$ (br.s; $m$-H).

$\mathrm{C}_{16} \mathrm{H}_{19} \mathrm{NO}_{5}(305.3)$ Ber. C 62.94 H 6.27 N 4.59 Gef. C 63.24 H 6.43 N 4.30

\section{Addukte von Nitriloxiden an 1,4-Cyclohexadien und Folgeprodukte}

9-Phenyl-7-oxa-8-aza-cis-bicyclo[4.3.0]nona-3,8-dien (25a): $3.20 \mathrm{ml}$ (23.2 mmol) Triethyl-

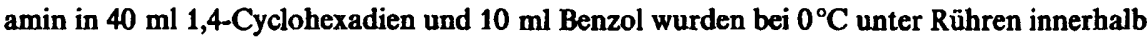
von $10 \mathrm{~h}$ mit $3.11 \mathrm{~g}(20.0 \mathrm{mmol})$ Benzhydroximoylchlorid ${ }^{25}$ in $16 \mathrm{ml}$ 1,4-Cyclohexadien und $4 \mathrm{ml}$ Benzol versetzt. Man lie $B$ auf $20^{\circ} \mathrm{C}$ kommen und gab nach einigen h soviel Wasser $\mathrm{zu}$, daß sich der Niederschlag auflöste. Man trennte die Phasen, extrahierte die wäßrige Phase mit Ether, trocknete die vereinigten organischen Phasen über $\mathrm{Na}_{2} \mathrm{SO}_{4}$ und engte i.Vak. ein. Es blieben $3.30 \mathrm{~g}(82 \%)$ eines gelben Ols zurück, das durch sein ${ }^{1} \mathrm{H}-\mathrm{NMR}$ Spektrum als weitgehend reines 25a identifiziert wurde. Das Analysenpräparat bereitete man durch Destillation, wobei die reine Substanz bei $120-130^{\circ} \mathrm{C}(\mathrm{Bad}) / 0.001$ Torr als farblose Flüssigkeit überging. - ' $\mathrm{H}-\mathrm{NMR}\left(\mathrm{CCl}_{4}\right): \delta=2.05,2.26$ und 2.34, 2.45 (jeweils ABSpektrum von $\mathrm{m}$ mit $J \approx 16 \mathrm{~Hz} ; 2-\mathrm{H}_{2}, 5-\mathrm{H}_{2}$ ), 3.64 (ddd, $J_{1,6}=9.7 \mathrm{~Hz}, J_{1,2}=5.6$ und 7.5 $\mathrm{Hz} ; 1-\mathrm{H}$ ), 4.80 (ddd, $J_{5,6}=3.5$ und $5.0 \mathrm{~Hz} ; 6-\mathrm{H}$ ), 5.78 (m; 3-, 4-H), 7.29 (m; 3 aromat. H), 7.57 (m; 2 aromat. $\mathrm{H})$.

$\mathrm{C}_{13} \mathrm{H}_{13} \mathrm{NO}(199.3)$ Ber. C 78.36 H 6.58 N 7.03 Gef. C 78.52 H 6.70 N 6.90

6,12-Diphenyl-4,10-dioxa-5,11-diaza-cis,cis,exo-tricyclo[7.3.0.0 $\left.0^{3.7}\right]$ dodeca-5,11-dien (26a) und 6,10-Diphenyl-4,12-dioxa-5,11-diaza-cis,cis,exo-tricyclo[ 7.3.0.0.0.7]dodeca-5,10-dien (28a): 
$\mathrm{Zu} 8.47 \mathrm{~g}$ (106 mmol) 1,4-Cyclohexadien in $10 \mathrm{ml}(72.5 \mathrm{mmol})$ Triethylamin tropfte man bei $60^{\circ} \mathrm{C}$ unter Rühren innerhalb einiger h $9.33 \mathrm{~g}(60.0 \mathrm{mmol})$ Benzhydroximoylchlorid in möglichst wenig Chloroform. Man arbeitete wie bei der Darstellung von 25a auf und erhielt nach Einengen i. Vak. $8.26 \mathrm{~g}$ Rückstand. Durch Chromatographie an Kieselgel (Akt.-St. I) mit Benzol trennte man das am schnellsten laufende Diphenylfuroxan ab; mit Benzol/Ether (95:5) kamen die weiteren Komponenten als Gemisch von der Säule. Durch Destillation bei $130-170^{\circ} \mathrm{C}(\mathrm{Bad}) / 0.001$ Torr trennte man daraus $300 \mathrm{mg}(2.5 \%) 25 \mathrm{a}$ ab. Aus dem Rückstand gewann man durch Chromatographie an Kieselgel (Akt.-St. I) mit Benzol/Ether (95:5) gelbe, nach mehrfachem Umlösen aus Ethanol oder Cyclohexan farblose Kristalle mit Schmp. $146-178^{\circ} \mathrm{C}$, die sich als 1.4:1-Gemisch aus $26 \mathrm{a}$ und $28 \mathrm{a}$ erwiesen. - ${ }^{1} \mathrm{H}-\mathrm{NMR}$ $\left(\mathrm{CDCl}_{3}\right): 26 \mathrm{a}: \delta=2.05\left(\mathrm{ddd}, J_{2,2}=14.5, J_{1,2 \mathrm{a}}=7.5, J_{2 \mathrm{a}, 3}=4.4 \mathrm{~Hz} ; 2-\mathrm{H}_{\mathrm{a}}\right), 2.17$ (ddd, $J_{1,2 \mathrm{~b}}=$ $\left.5.7, J_{2 \mathrm{~b}, 3}=7.2 \mathrm{~Hz} ; 2-\mathrm{H}_{\mathrm{b}}\right), 3.99$ (ddd, $J_{1,9}=11.4 \mathrm{~Hz} ; 1-\mathrm{H}$ ), 4.81 (ddd; 3-H), $\mathrm{C}_{6} \mathrm{H}_{5}: 7.32-7.45$ (m; $m-, p-H), 7.67(\mathrm{~m} ; o-\mathrm{H}) ; 28 \mathrm{a}: \delta=1.86\left(\mathrm{t}, J_{7,8}+J_{7,8^{\prime}}=13.0 \mathrm{~Hz} ; 8-\mathrm{H}_{2}\right), 2.43\left(\mathrm{t}, J_{1,2}+\right.$ $\left.J_{1,2^{\prime}}=11.2 \mathrm{~Hz} ; 2-\mathrm{H}_{2}\right), 3.67\left(\mathrm{dt}, J_{3,7}=10.4 \mathrm{~Hz} ; 7-\mathrm{H}\right), 4.98(\mathrm{dt} ; 1-\mathrm{H}), \mathrm{C}_{6} \mathrm{H}_{5}: 7.32-7.45(\mathrm{~m} ; \mathrm{m}-$, p-H), $7.53(\mathrm{~m} ; o-\mathrm{H}) .-{ }^{13} \mathrm{C}-\mathrm{NMR}\left(\mathrm{CDCl}_{3}\right): 26 \mathrm{a}: \delta=25.02$ (t; C-2), 42.20 (d; C-1), 77.68 (d; $\mathrm{C}-3$ ), 158.48 (s; C-6), $\mathrm{C}_{6} \mathrm{H}_{5}: 126.97$ und 128.95 (jeweils d; $o-, m-\mathrm{C}$ ), 128.66 (s; ipso-C), 130.21 (d; $p$-C); 28a: 21.65 und 26.97 (jeweils t; C-2, -8), 43.59 (d; C-7), 77.68 (d; C-1), 158.68 (s; C6), $\mathrm{C}_{6} \mathrm{H}_{5}: 126.78$ und 128.95 (jeweils d; $o-, m-\mathrm{C}$ ), 128.58 (s; ipso-C), 130.16 (d; $p$-C).

$\mathrm{C}_{20} \mathrm{H}_{18} \mathrm{~N}_{2} \mathrm{O}_{2}$ (318.4) Ber. C 75.45 H 5.70 N 8.80 Gef. C 75.31 H 5.75 N 8.77

9-(2,4,6-Trimethylphenyl)-7-oxa-8-aza-cis-bicyclo[4.3.0]nona-3,8-dien (25b), 6,12-Bis(2,4,6-trimethylphenyl)-4,10-dioxa-5,11-diaza-cis,cis,exo-tricyclo[7.3.0.0.7.7dodeca-5,11-dien (26b) und -cis,cis,endo-tricyclo/7.3.0.0.7 Jdodeca-5,11-dien (27b): Aus dem Gemisch von 2,4,6Trimethylbenzonitriloxid ${ }^{9}$ und 1,4-Cyclohexadien im Verhältnis $1: 2$ gingen nach mehreren Tagen bei $20^{\circ} \mathrm{C} \mathrm{25b}$, 26b und 27b hervor. Reines 25b erhielt man durch Destillation bei $140-160^{\circ} \mathrm{C}(\mathrm{Bad}) / 0.001$ Torr als farbloses $\mathrm{Ol}$, das nach einiger Zeit zu Kristallen mit Schmp. $44-48^{\circ} \mathrm{C}$, nach Umlösen aus Ethanol/Hexan $48-49^{\circ} \mathrm{C}$, erstarrte. - ${ }^{1} \mathrm{H}-\mathrm{NMR}\left(\mathrm{CDCl}_{3}\right.$ ): $\delta=1.85-2.50\left(\mathrm{~m} ; 2-\mathrm{H}_{2}, 5-\mathrm{H}_{2}\right), 2.24$ (br.s; $3 \mathrm{CH}_{3}$ ), 3.76 (ddd, $J_{1.6}=11.0 \mathrm{~Hz}, \Sigma J_{1,2}=11.0$ $\mathrm{Hz} ; 1-\mathrm{H}$ ), 5.00 (dt, $\Sigma J_{s, 6}=9.2 \mathrm{~Hz} ; 6-\mathrm{H}$ ), 5.85 (m; 3-, 4-H), 6.87 (br.s; 2 aromat. $H$ ).

$\mathrm{C}_{16} \mathrm{H}_{19} \mathrm{NO}$ (241.3) $\quad$ Ber. C 79.63 H 7.94 N 5.80 Gef. C 79.93 H 8.11 N 5.71

Aus dem Rückstand obiger Destillation erhielt man durch mehrfaches Umlösen aus Ethanol ein 6.5:1-Gemisch aus $26 \mathrm{~b}$ und $27 \mathrm{~b}$ mit Schmp. $269-273^{\circ} \mathrm{C}$. - ${ }^{1} \mathrm{H}-\mathrm{NMR}\left(\mathrm{CDCl}_{3}\right)$ : $26 \mathrm{~b}$ : $\delta=1.81\left(\mathrm{ddd}, J_{2,2}=14.3, J_{1,2 \mathrm{a}}=7.0, J_{2 \mathrm{a}, 3}=4.3 \mathrm{~Hz} ; 2-\mathrm{H}_{\mathrm{a}}\right), 1.91\left(\approx \mathrm{dt}, J_{1,2 \mathrm{~b}}=6.9, J_{2 \mathrm{~b}, 3}=\right.$ $7.4 \mathrm{~Hz} ; 2-\mathrm{H}_{\mathrm{b}}$ ), 2.29 (s; $3 \mathrm{CH}_{3}$ ), 3.86 (dt, $\left.J_{1,9}=11.0 \mathrm{~Hz} ; 1-\mathrm{H}\right), 4.90$ (ddd; 3-H), 6.91 (br.s; 2 aromat. H); $27 \mathrm{~b}$ : $\delta=1.79$ (weitgehend überlagert durch ein Signal von $26 \mathrm{~b} ; 2-\mathrm{H}_{\mathrm{a}}$ ), 2.04 $\left(\mathrm{dt} ; J_{2,2}=12.9, J_{1,2 b}=5.6, J_{2 b, 3}=6.0 \mathrm{~Hz} ; 2-\mathrm{H}_{\mathrm{b}}\right), 2.26\left(\mathrm{~s} ; 3 \mathrm{CH}_{3}\right), 3.55$ (ddd, $J_{1,2 \mathrm{a}}=13.6$, $\left.J_{1,9}=10.9 \mathrm{~Hz} ; 1-\mathrm{H}\right), 4.73$ (dt, $\left.J_{2 \mathrm{a}, 3}=11.1 \mathrm{~Hz} ; 3-\mathrm{H}\right), 6.90$ (br.s; 2 aromat. H). $-{ }^{13} \mathrm{C}-\mathrm{NMR}$ $\left(\mathrm{CDCl}_{3}\right): 26 \mathrm{~b}: \delta=20.26$ (q; $o-\mathrm{CH}_{3}$ ), 21.02 (q; $p-\mathrm{CH}_{3}$ ), 23.63 (t; C-2), 46.25 (d; C-1), 76.59 (d; C-3), 125.06 (s; ipso-C), 129.06 (d; m-C), 136.79 (s; o-C), 138.98 (s; p-C), 159.51 (s; C-6); 27b: $\delta=20.05$ (q; o- $\mathrm{CH}_{3}$ ), 24.57 (t; C-2), 47.95 (d; C-1), 77.41 (d; C-3), 128.85 (d; m-C), 158.42 (s; C-6); die fehlenden Signale von $27 \mathrm{~b}$ fielen entweder mit denen von $26 \mathrm{~b}$ zusammen oder waren wegen $\mathrm{zu}$ geringer Intensität nicht auffindbar.

$\mathrm{C}_{26} \mathrm{H}_{30} \mathrm{~N}_{2} \mathrm{O}_{2}$ (402.5) Ber. C 77.58 H 7.51 N 6.96 Gef. C 77.63 H 7.47 N 6.99

Phenyl-(2,4,6-trimethylphenyl)-ketimin (31): Beim Erhitzen auf $200-230^{\circ} \mathrm{C}$ in Substanz wandelte sich $25 \mathrm{~b}$ in das bekannte ${ }^{21)} 31 \mathrm{um}$, das durch Destillation bei $125-130^{\circ} \mathrm{C} \mathrm{(Bad)/}$ 0.001 Torr rein erhalten wurde. Die Identifizierung erfolgte anhand der Elementaranalyse und der Spektren. - IR (Film): 3230 (N-H), 1610, $1570 \mathrm{~cm}^{-1}(\mathrm{C}=\mathrm{N}, \mathrm{C}=\mathrm{C})$. - ${ }^{1} \mathrm{H}-\mathrm{NMR}$ $\left(\mathrm{CCl}_{4}\right): \delta=2.03$ (s; o- $\left.\mathrm{CH}_{3}\right), 2.27$ (s; $\left.p-\mathrm{CH}_{3}\right), 6.80$ (br.s; 2 Mesityl-H), 7.32 (m; 3 Phenyl-H), 7.68 (m; 2 Phenyl-H), 9.2 (sehr breit; NH).

Chem. Ber. 118 (1985) 
Die Umsetzung mit Acetanhydrid erbrachte das $N$-Acetyl-Derivat mit Schmp. $92.5-93^{\circ} \mathrm{C}$ (Lit. ${ }^{21)}$ 93.5-94 $\left.{ }^{\circ} \mathrm{C}\right)$. - IR (KBr): $1675(\mathrm{C}=\mathrm{O}), 1615 \mathrm{~cm}^{-1}(\mathrm{C}=\mathrm{N})$.

trans-3,4-Dibrom-9-phenyl-7-oxa-8-aza-cis-bicyclo[4.3.0/non-8-ene (32 und 33): $\mathrm{Zu} 1.60 \mathrm{~g}$ $(8.03 \mathrm{mmol}) 25 \mathrm{a}$ in $20 \mathrm{ml}$ absol. Tetrachlormethan tropfte man bei $0^{\circ} \mathrm{C}$ unter Rühren innerhalb von $45 \mathrm{~min} 1.30 \mathrm{~g}(8.15 \mathrm{mmol})$ Brom in $10 \mathrm{ml}$ absol. Tetrachlormethan. Einengen i. Vak. ergab $2.74 \mathrm{~g}(95 \%)$ eines gelben Öls, dessen ${ }^{1} \mathrm{H}$-NMR-Spektrum das Vorliegen der Stereoisomeren 32 und 33 im Verhāltnis 3:1 anzeigte. Die Behandlung des Rohprodukts mit Ether führte zur Bildung von $1.15 \mathrm{~g}(40 \%)$ Kristallen, nach Umlösen aus Ethanol mit Schmp. $119-121^{\circ} \mathrm{C}$, die sich als reines Überschußisomeres 32 erwiesen. - MS (70 eV): $m / e=361,359,357\left(44 \%, 91 \%, 46 \%, \mathrm{M}^{+}\right), 77(100)$. ${ }^{1} \mathrm{H}-\mathrm{NMR}\left(\mathrm{CDCl}_{3}\right): \delta=2.28$ (ddd, $\left.J_{1,2 \mathrm{~b}}=6.9, J_{2 \mathrm{a}, 2 \mathrm{~b}}=14.9, J_{2 \mathrm{~b}, 3}=3.1 \mathrm{~Hz} ; 2-\mathrm{H}_{\mathrm{b}}\right), 2.46\left(\mathrm{ddd}, J_{1,2 \mathrm{a}}=11.0, J_{2 \mathrm{a}, 3}=3.2 \mathrm{~Hz} ; 2-\mathrm{H}_{\mathrm{a}}\right.$ ), $2.80\left(\mathrm{dt}, J_{4,5 \mathrm{a}}=J_{5 \mathrm{a}, 6}=2.5, J_{5 \mathrm{a}, 5 \mathrm{~b}}=17.0 \mathrm{~Hz} ; 5-\mathrm{H}_{\mathrm{a}}\right), 2.97\left(\mathrm{dt}, J_{4,5 \mathrm{~b}}=J_{5 \mathrm{~b}, 6}=5.0 \mathrm{~Hz} ; 5-\mathrm{H}_{\mathrm{b}}\right)$, $3.72\left(\mathrm{dt}, J_{1.6}=6.9 \mathrm{~Hz} ; 1-\mathrm{H}\right), 4.53-4.60(\mathrm{~m} ; 4,6-\mathrm{H}), 4.67\left(\approx \mathrm{q}, J_{3,4}=3.9 \mathrm{~Hz} ; 3-\mathrm{H}\right), 7.43(\mathrm{~m}$; 3 aromat. H), 7.73 (m; 2 aromat. H). $-{ }^{13} \mathrm{C}-\mathrm{NMR}\left(\mathrm{CDCl}_{3}\right): \delta=28.54$ (t, Doppelsignal; C-2, -5), 40.92 (d; C-1), 44.86 und 49.92 (jeweils d; C-3, -4), 77.71 (d; C-6), 162.36 (s; C-9), $\mathrm{C}_{6} \mathrm{H}_{\mathrm{s}}$ : 126.97 und 129.00 (jeweils d; $o-, m-\mathrm{C}$ ), 128.76 (s; ipso-C), 130.42 (d; $p$-C).

$$
\begin{array}{lllllll}
\mathrm{C}_{13} \mathrm{H}_{13} \mathrm{Br}_{2} \mathrm{NO}(359.1) & \text { Ber. C } 43.48 & \mathrm{H} 3.65 & \mathrm{Br} 44.51 & \mathrm{~N} 3.90 \\
& \text { Gef. C } 43.79 & \mathrm{H} 3.79 & \mathrm{Br} & 44.29 & \mathrm{~N} 3.52
\end{array}
$$

UnterschuBisomeres 33: 'H-NMR $\left(\mathrm{CDCl}_{3}\right): \delta=1.98\left(\mathrm{dt}, J_{1,2 \mathrm{a}}=J_{2 a, 3}=11.0, J_{2 \mathrm{a}, 2 \mathrm{~b}}=\right.$ $14.0 \mathrm{~Hz} ; 2-\mathrm{H}_{\mathrm{a}}$ ), 2.42 (ddd, $J_{5 \mathrm{~b}, 6} \approx 4.5 \mathrm{~Hz} ; 5-\mathrm{H}_{\mathrm{b}}$ ), 2.76 (ddd; $2-\mathrm{H}_{\mathrm{b}}$ ), 3.00 (ddd, $J_{4.5 \mathrm{a}}=4.5$, $\left.J_{5 \mathrm{a}, 5 \mathrm{~b}}=15.3, J_{5 \mathrm{a}, 6}=3.0 \mathrm{~Hz} ; 5-\mathrm{H}_{\mathrm{a}}\right), 3.50\left(\mathrm{dt}, J_{1,2 \mathrm{~b}}=J_{1,6}=6.8 \mathrm{~Hz} ; 1 . \mathrm{H}\right), 4.12$ (ddd, $J_{3,4}=$ 9.5, $\left.J_{4,5 \mathrm{~b}}=11.5 \mathrm{~Hz} ; 4-\mathrm{H}\right), 4.33\left(\mathrm{ddd}, J_{2 \mathrm{~b}, 3}=4.4 \mathrm{~Hz} ; 3-\mathrm{H}\right), \approx 4.55(\mathrm{~m} ; 6-\mathrm{H}), 7.4-7.8(\mathrm{~m}$; $\mathrm{C}_{6} \mathrm{H}_{3}$ ). $-{ }^{13} \mathrm{C}-\mathrm{NMR}\left(\mathrm{CDCl}_{3}\right): \delta=35.80$ und 36.17 (jeweils $\mathrm{t}$; C-2, -5 ), 44.87 (d; C-1), 51.00 und 51.98 (jeweils d; C-3, -4), 80.18 (d; C-6), 162.74 (s; C-9); wegen der Signale von Verunreinigungen konnten die $\mathrm{C}_{6} \mathrm{H}_{5}$-Linien nicht identifiziert werden.

Reaktion von 32 mit 1,8-Diazabicyclo[5.4.0]undec-7-en (DBU): $\mathrm{Zu} 55 \mathrm{mg}(0.153 \mathrm{mmol})$ 32 in $0.5 \mathrm{ml}$ absol. Tetrahydrofuran gab man bei $20^{\circ} \mathrm{C} 46.6 \mathrm{mg}(0.306 \mathrm{mmol}) \mathrm{DBU}$. Es zeigte sich alsbald eine Abscheidung farbloser Kristalle (wahrscheinlich DBU - HBr), die sich im Laufe von $24 \mathrm{~h}$ verstärkte. Gelegentlich aufgenommene 'H-NMR-Spektren belegten die fortschreitende Abnahme der 32-Resonanzen. Im Bereich der Signale aromatischer Protonen wuchs ein Singulett an, wahrscheinlich von Benzol verursacht. Wenig intensive Signale bei $\delta=6.1$ und 6.3 könnten von $1 \mathrm{a}$ oder seinem Vorläufer nach Eliminierung von einem Molekül $\mathrm{HBr}$ aus 32 hergerührt haben. Nach Filtration des Gemisches und Einengen des Filtrats i.Vak. wurden durch Chromatographie an Kieselgel (Säule $20 \times 1 \mathrm{~cm}$ ) mit Toluol als Laufmittel $10.5 \mathrm{mg}(57 \%)$ Diphenylfuroxan (35) isoliert und durch Vergleich seines IRSpektrums mit dem eines authentischen Präparats identifiziert.

9-Phenyl-7-oxa-8-azatricyclo[4.3.0.0 $0^{13}$ /nona-4,8-dien (36): Unter Stickstoff tropfte man $190 \mathrm{mg}(0.53 \mathrm{mmol}) 32$ in $2 \mathrm{ml}$ absol. Tetrahydrofuran innerhalb von $30 \mathrm{~min} \mathrm{zu} 149 \mathrm{mg}$ (1.33 mmol) Kalium-tert-butoxid in $2 \mathrm{ml}$ Tetrahydrofuran, die bei $-35^{\circ} \mathrm{C}$ gerührt wurden. Dann rührte man das dunkelrote Gemisch $1 \mathrm{~h}$ bei $-35^{\circ} \mathrm{C}$ und $2 \mathrm{~h}$ bei $20^{\circ} \mathrm{C}$, wobei Farbänderung nach Gelb eintrat. Man goß auf Eis/Wasser, extrahierte mit Pentan, wusch die organische Phase mit Wasser und trocknete sie über $\mathrm{Na}_{2} \mathrm{SO}_{4}$. Verdampfen der Lösungsmittel i. Vak. hinterließ ein gelbes Ö, aus dem durch Zugabe von Pentan $65 \mathrm{mg}$ farblose Kristalle mit Schmp. $75-76^{\circ} \mathrm{C}$ fielen. Präparative Schichtchromatographie (Chromatotron) auf Kieselgel mit Ether/Pentan (1:3) erbrachte $30.3 \mathrm{mg}(29 \%) 36 \mathrm{mit} \mathrm{Schmp.} 88^{\circ} \mathrm{C}$. - MS (70 eV): $m / e=197\left(17 \%, \mathrm{M}^{+}\right), 66(100) .-{ }^{1} \mathrm{H}-\mathrm{NMR}:$ Tab. $4 .-{ }^{13} \mathrm{C}-\mathrm{NMR}\left(\mathrm{CDCl}_{3}\right): \delta=25.50(\mathrm{t}$; 
C-2), 33.36 (d; C-3), 41.16 (s; C-1), 92.65 (d; C-6), 130.84 und 139.62 (jeweils d; C-4, -5), 157.66 (s; C-9), $\mathrm{C}_{6} \mathrm{H}_{5}: 126.87$ und 128.79 (jeweils d; $0-, m-\mathrm{C}$ ), 129.15 (s; ipso-C), 129.87 (d; $p-\mathrm{C}$ ).

$\mathrm{C}_{13} \mathrm{H}_{11} \mathrm{NO}(197.2)$ Ber. C 79.16 H 5.62 N 7.10 Gef. C 78.96 H 5.62 N 6.78

1) Aus der Diplomarbeit und der Dissertation B. Mattauch, Univ. Würzburg 1981 und 1985.

2) L. A. Paquette und R. J. Haluska. J. Am. Chem. Soc. 94, 534 (1972).

3) $G$. Brüntrup und $M$. Christl, Tetrahedron Lett. 1973, 3369.

4) W. J. Linn und R. E. Benson, J. Am. Chem. Soc. 87, 3657 (1965).

5) L. A. Paquette, Synthesis 1975, 347, und zit. Lit.; P. Bischof, R. Gleiter, R. T. Taylor, A. R. Browne und L. A. Paquette, J. Org. Chem. 43, 2391 (1978), und zit. Lit.; M. Christl, G. Freitag und $G$. Brüntrup, Chem. Ber. 111, 2307, 2320 (1978).

6) M. Christl, Angew. Chem. 85, 666 (1973); Angew. Chem., Int. Ed. Engl. 12, 660 (1973).

7) H.-D. Martin und M. Hekman, Angew. Chem. 88, 447 (1976); Angew. Chem., Int. Ed. Engl. 15, 431 (1976): M. J. Goldstein, R. S. Leight und M. S. Lipton. J. Am. Chem. Soc. 98, 5717 (1976).

8) 8a) E. E. van Tamelen und S. P. Pappas, J. Am. Chem. Soc. 85, 3297 (1963). - ${ }^{86)}$ E. E. van Tamelen, S. P. Pappas und K. L. Kirk, J. Am. Chem. Soc. 93, 6092 (1971). - ${ }^{80)}$ E. E. van Tamelen und D. Carty, J. Am. Chem. Soc. 93, 6102 (1971).

9) C. Grundmann und J. M. Dean, J. Org. Chem. 30, 2809 (1965).

${ }^{10)}$ R. Huisgen und W. Mack, Tetrahedron Lett. 1961, 583; Chem. Ber. 105, 2805 (1972).

11) R. Huisgen, M. Seidel, G. Wallbillich und H. Knupfer, Tetrahedron 17, 3 (1962).

12) L. Horner und H. Merz, Liebigs Ann. Chem. 570, 89 (1950).

13) M. S. Raasch, J. Org. Chem. 45, 856 (1980).

14) M. Christl, H. Leininger und P. Kemmer, Chem. Ber. 117, 2963 (1984).

15) R. A. Sheldon und J. K. Kochi in Organic Reactions, Bd. 19, S. 279, John Wiley und Sons, New York, London, Sidney, Toronto 1972.

16) ${ }^{162)}$ B. M. Trost und R.M. Cory. J. Am. Chem. Soc. 93, 5573 (1971). - ${ }^{16 b)}$ T. J. Katz und N. Acton, J. Am. Chem. Soc. 95, 2738 (1973). - 160) N. J. Turro, C. A. Renner, W. H. Waddell und T. J. Katz, J. Am. Chem. Soc. 98, 4320 (1976). - 160) B. M. Trost, P. H. Scudder, R.M. Cory, N. J. Turro, V. Ramamurthy und T. J. Katz, J. Org. Chem. 44, 1264 (1979). - ${ }^{16 e)}$ N. J. Turro und V. Ramamurthy, Rec. Trav. Chim. Pays-Bas 98, 173 (1979).

i7 B. M. Trost und R. M. Cory, J. Am. Chem. Soc. 93, 5572 (1971); B. M. Trost, R. M. Cory, P. H. Scudder und H. B. Neubold, cbenda 95, 7813 (1973).

18) R. Sustmann, R. Huisgen und H. Huber, Chem. Ber. 100, 1802 (1967).

19) C. Grundmann und P. Grünanger, The Nitrile Oxides, Springer Verlag, Berlin 1971.

20) K. Bast, M. Christl, R. Huisgen und W. Mack, Chem. Ber. 106, 3312 (1973).

${ }^{21)}$ R. C. Fuson, W. D. Emmons und J. P. Freeman, J. Am. Chem. Soc. 75, 5321 (1953).

22) T. G. Burrowes, W. R. Jackson, S. Faulks und I. Sharp, Aust. J. Chem. 30, 1855 (1977); H. Grund und V. Jäger, J. Chem. Res. (S) 1979, 54.

23) 23a) $M$. Christl, $E$. Brunn und $F$. Lanzendörfer, J. Am. Chem. Soc. 106, 373 (1984). - ${ }^{23 b)} H$. Prinzbach, H. Hagemann, J. H. Hartenstein und R. Kitzing, Chem. Ber. 98, 2201 (1965); M. Barfield und S. Sternhell, J. Am. Chem. Soc. 94, 1905 (1972).

24) $R$. N. McDonald und C. E. Reineke, J. Org. Chem. 32, 1878 (1967)

25) A. Werner und H. Buss, Ber. Dtsch. Chem. Ges. 27, 2193 (1894); K.-C. Liu, B. R. Shelton und R. K. Howe, J. Org. Chem. 45, 3916 (1980).

${ }^{26)} T$. J. Katz, E. J. Wang und N. Acton, J. Am. Chem. Soc. 93, 3782 (1971); T. J. Katz, R. J. Roth, N. Acton und E. J. Carnahan, Org. Synth. 53, 157 (1973).

27) Zur Darstellung von Benzvalen verwendeten wir bromidfreies Methyllithium von CHEMETALL, Frankfurt am Main.

28) M. Christl, H. Leininger und B. Mattauch, Spectrosc. Int. J. 2, 184 (1983). 